\title{
Dental Bulk-Fill Resin Composites Polymerization Efficiency: A Systematic Review and Meta-Analysis
}

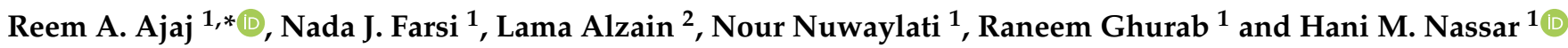 \\ 1 Faculty of Dentistry, King Abdulaziz University, Jeddah 21589, Saudi Arabia; njfarsi@kau.edu.sa (N.J.F.); \\ nnuwaylati@stu.kau.edu.sa (N.N.); rghurab0001@stu.kau.edu.sa (R.G.); hnassar@kau.edu.sa (H.M.N.) \\ 2 Ministry of Health, Jeddah 21589, Saudi Arabia; lalzain@moh.gov.sa \\ * Correspondence: raajaj@kau.edu.sa
}

Citation: Ajaj, R.A.; Farsi, N.J.;

Alzain, L.; Nuwaylati, N.; Ghurab, R.;

Nassar, H.M. Dental Bulk-Fill Resin

Composites Polymerization

Efficiency: A Systematic Review and

Meta-Analysis. J. Compos. Sci. 2021, 5

149. https://doi.org/10.3390/

jcs5060149

Academic Editor: Masao Irie

Received: 23 April 2021

Accepted: 30 May 2021

Published: 2 June 2021

Publisher's Note: MDPI stays neutral with regard to jurisdictional claims in published maps and institutional affiliations.

Copyright: (c) 2021 by the authors. Licensee MDPI, Basel, Switzerland. This article is an open access article distributed under the terms and conditions of the Creative Commons Attribution (CC BY) license (https:// creativecommons.org/licenses/by/ $4.0 /)$.

\begin{abstract}
Dental Bulk-Fill Composites (BFCs) and Bulk-Fill Flowables (BFFs) were introduced in the market to facilitate efficient bulk filling of cavities up to $5 \mathrm{~mm}$. The aim of this study was to synthesize the literature investigating their polymerization efficiency. A comprehensive search of PubMed and the Cochrane Library from 2010 to January 2019 was performed using the medical subject headings. Screening of the titles, abstracts and full text was performed. Data extraction for relevant information was done on the included studies. Clinically relevant parameters were selected to present the study estimates (meta-analysis) using a random effects model for polymerization efficiency (Degree of Conversion (DC) and Depth of Cure (DoC)). Twenty one studies fulfilled the inclusion criteria and were included in the analysis reporting seven BFCs and nine BFFs. Ten materials reported acceptable DC values of above $55 \%$ and ten materials reported adequate DoC values. Most of the stated materials reported adequate DC and DoC values in at least one investigation with BFFs showing higher and more acceptable values compared to packable BFCs. It is suggested that future studies be carried out using a standard methodology following the ISO 4049 standard and manufacturer's instructions to compare results.
\end{abstract}

Keywords: bulk-fill composite; bulk-fill flowable; polymerization efficiency; degree of conversion; depth of cure

\section{Introduction}

Photo-polymerizable Resin-Based Composites (RBCs) have always been preferred as the material of choice by clinicians due to superior esthetics, improved properties, and operator-controlled working time [1-3]. The application process of these materials requires placing the material in increments no greater than $2 \mathrm{~mm}$ in thickness in order to ensure proper penetration of the curing light to the deeper parts of the restoration warranting optimum polymerization [4,5]. Additionally, filling cavities in an incremental manner reduces the magnitude of volumetric shrinkage stresses which accompany the polymerization reaction [6,7]. However, the incremental-placement method is time-consuming, technique sensitive, and depending on the clinician's experience may introduce unwanted voids that could weaken the resulting restoration $[4,8,9]$.

Consequently, new light-cure composite formulations were introduced in the market in order to facilitate bulk filling of cavities where the manufacturers claim that these materials can fill up to $5 \mathrm{~mm}$ with predictable polymerization efficiency [4] without the need for the incremental technique; hence expediting the restorative procedure $[10,11]$. These materials, termed Bulk-Fill Composites (BFCs), have different chemistries which facilitate deeper penetration of the radiated energy from light-cure devices. These materials contain more sensitive photoinitiators other than the commonly used camphorquinone initiator used in conventional RBCs. In addition, these materials tend to be more translucent which allows for more light to pass through $[12,13]$. These modifications would ensure better exposure of the deeper parts of the restoration to the curing light leading to deeper 
depth of cure $(\mathrm{DoC})$ and facilitating more conversion of monomers into polymers leading to better degree of conversion (DC).

Further modifications of the filler contents led to the introduction of low viscosity BFCs which can be utilized as cavity liners while maintaining the bulk-fill potential of their high-viscosity counterparts. These Bulk-Fill Flowables (BFFs) have higher resin matrix content which allows for better stress distribution during the polymerization reaction and consequently less overall stress [14,15].

However, polymerization efficiency of BFCs and BFFs must be tested either directly or indirectly to ensure favorable clinical service. Direct methods, such as Fourier-Transform Infrared Spectroscopy (FTIR) and Raman Spectroscopy (RS), measure the DC by determining the proportion of the remaining concentration of the aliphatic $\mathrm{C}=\mathrm{C}$ double bonds in a cured sample relative to the total number of $C=C$ bonds in the uncured material $[16,17]$ which indicates the conversion ratio of double bonds $(C=C)$ into single bonds $(C-C)$ during the propagation phase of the polymerization reaction $[18,19]$. This is an important parameter in determining the final physical, mechanical, and biological properties of photo-activated resin composites. The DC of resin composites is influenced by the spectral distribution and intensity of the curing light as well as the shade, opacity, and chemical composition of the RBC [20]. It has been established that BFF materials can reach a clinically acceptable DC, although some of them are on the lower limit of $\sim 55 \%$ monomer-to-polymer conversion ratio [21]. Indirect methods can be used to judge the polymerization efficiency by determining the DoC using the Scrapping Method (SM) or Microhardness Ratio (MHR) [22-25]. The former assesses the remaining thickness, which is the length of the cylindrical specimen of cured resin composite, after removal of the uncured resin composite with a plastic spatula, divided by two [24]. While the microhardness (Vickers and Knoop) assesses the bottom-to-top hardness ratio $[22,24,26]$.

In the literature, numerous studies explored and compared the polymerization reaction and their results were inconsistent. Thus, it is paramount to examine the current available evidence regarding BFCs and BFFs formulations in relation to their DC and DoC. The primary aim of this study was to synthesize the literature for publications investigating the polymerization efficiency of BFCs and BFFs using all available direct (RS and FTIR) and indirect methods (SM and MHR). Further, we aim to statistically combine the estimates of $\mathrm{DC}$ and DoC to provide clinically relevant outcome values for polymerization efficiency.

\section{Materials and Methods}

This systematic review was done following the Preferred Reporting Items for Systematic Reviews and Meta-Analysis (PRISMA) guidelines [27].

\subsection{Search Strategy}

A comprehensive search of NCBI (PubMed) and the Cochrane Library from January 2010 to January 2019 was performed. The following medical subject headings (MeSH terms) were used: "polymerization efficiency", "resin composites", "bulk fill composites", "depth of cure" and "degree of conversion". The following search sequences was applied in both search engines: (1) "polymerization efficiency AND resin composites", (2) "bulk fill composites AND depth of cure", (3) "bulk fill composites AND degree of conversion", (4) "polymerization efficiency OR degree of conversion". Additionally, references of relevant retrieved reviews were screened for other potential eligible articles.

\subsection{Inclusion and Exclusion Criteria}

All English language articles were included and the inclusion criteria used were (a) utilize in-vitro methods, (b) studies documenting polymerization efficiency of BFC by measuring selected parameters: DoC or DC, and (c) cavities with a minimum depth of $3 \mathrm{~mm}$. Only studies utilizing in vitro methodology were included due to the difficulty in standardization of the in vivo studies and to reduce the independent variables that affect the outcome of the studies. Studies with a minimum depth of $3 \mathrm{~mm}$ were included to 
be in line with the objective of using BFCs which is curing beyond the standard $2 \mathrm{~mm}$ incremental fill conventional resin composites [4]. All non-English language articles as well as reviews were excluded. Articles investigating different parameters than DC and DoC or reporting values other than the numerical mean were also excluded.

\subsection{Study Selection}

All retrieved studies were screened by two independent reviewers (R.A.G and N.A.N) for eligibility. Disagreement was resolved by a third reviewer (R.A.A). In the first step of screening, titles were screened, and completely irrelevant studies were excluded. In the second screening stage, abstracts were screened and studies not investigating relevant outcomes (DoC and DC), and in vivo conducted studies were excluded. Subsequently, full-text articles that were potentially acceptable were assessed to identify the final set of eligible articles, as shown in Figure 1.

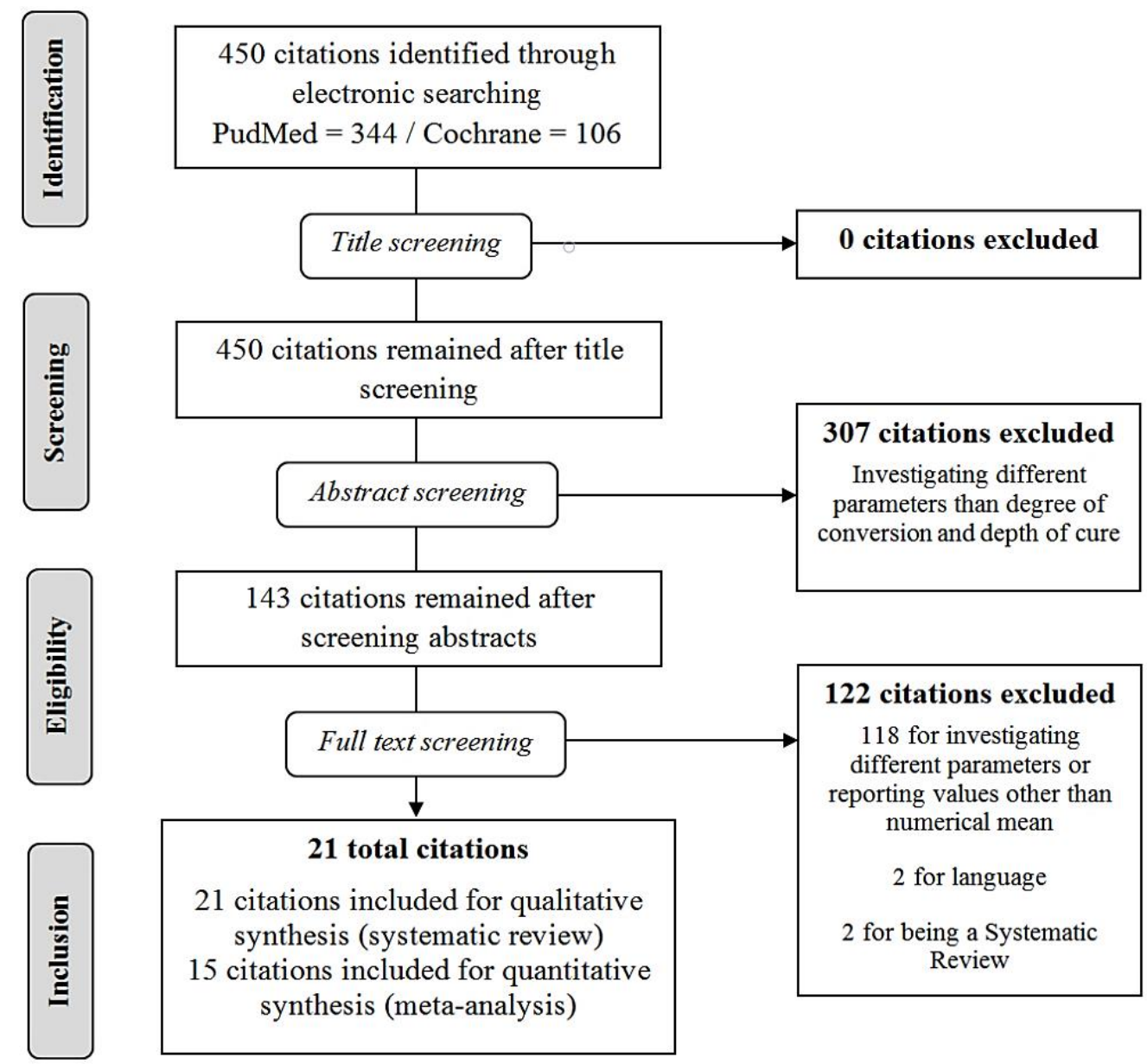

Figure 1. A PRISMA flow chart illustrating the identification process of the included studies in the systematic review and meta-analysis.

\subsection{Risk of Bias}

The quality of the studies was assessed for risk of bias by N.A.N, using a list of questions that were generated by the authors, assessing the pertinent objective of the study, methodology used (sample size and presence of a control group), and the measured outcomes.

\subsection{Data Extraction}

From each of the included studies, the following information was extracted: first author name, brand of the composite, type of material (BFC or BFF), sample size, method of measuring polymerization shrinkage, light curing type, light curing time and its intensity. 
Different outcomes were extracted for each method. For the SM, the remaining thickness was reported; while for Knoop and Vickers microhardness, microhardness values at the top and at different depths $(3,4,5$, and $6 \mathrm{~mm})$ were recorded to report the bottom-totop MHR. For RS and FTIR, the DC at the top surface, and at different depths ( 3 and $4 \mathrm{~mm}$ ) and at different time points $(0,24 \mathrm{~h}$ and 1 week) were recorded.

A customized table was designed for the data extraction. The table was divided into four groups according to different methodology: FTIR, RS, SM, and MHR. The data extraction was done independently by two reviewers (L.M.A and R.A.G), and any discrepancies were solved by consulting other authors (H.M.N or R.A.A). When required information was missing or not clear in the retrieved papers, authors of the relevant papers were contacted.

\subsection{Summary Measures}

The outcomes of interest were: the DC which was measured directly through RS and FTIR, and the DoC which was measured indirectly via SM and bottom-to-top MHR obtained from Vickers and Knoop microhardness tests. DC values range from 0-100\%. DC values $\approx 55 \%$ and above were considered acceptable according to AlShali and Silikas 2013 [21]. Remaining thickness values of $3.5 \mathrm{~mm}$ and above were considered acceptable for the SM according to ISO 4049 as this value is not more than $0.5 \mathrm{~mm}$ below the value of at least $4 \mathrm{~mm}$ that is stated by the manufacturers of the BFCs and BFFs as the depth with sufficient polymerization [28]. The bottom-to-top MHR values of at least 0.8 were considered as an acceptable ratio according to Poggio et al. 2012 [3].

\subsection{Statistical Analyses}

Qualitative synthesis of the extracted outcome data was done. The following information was presented for the materials that assessed polymerization sufficiency with the direct methods: material type (BFC or BFF), light cure time (10, 20, 25, 30 and $40 \mathrm{~s})$, DC (mean/SD) for the following time points: immediate cure (at top and $4 \mathrm{~mm}$ depth), $24 \mathrm{~h}$ (at top and $4 \mathrm{~mm}$ depth), 7 days (at 3 and $4 \mathrm{~mm}$ depth), and 30 days (at $4 \mathrm{~mm}$ depth). For the indirect methods the following parameters were presented; for the SM: light cure time (10, 20, 30 and $40 \mathrm{~s}$ ), and remaining thickness in $\mathrm{mm}$ (mean/SD). While for the microhardness, light cure time (10, 20, 30, 40, and 60 s), hardness number (Vickers or Knoop; mean/SD), and bottom-to-top MHR (ratio), at depths 3, 4, 5, and $6 \mathrm{~mm}$, were presented. Studies reported performing the test immediately, $5 \mathrm{~min}$, or $15 \mathrm{~min}$ after light-curing were grouped under zero hours. Additionally, studies with unreported testing time were assumed to have done immediate testing and were placed with the " $0 \mathrm{~h}$ " category.

To quantitatively summarize the estimates of the different studies, specific fixed parameters were selected according to the ISO standards [28] to present the study estimates. Thus, only samples light cured for the standard $20 \mathrm{~s}$ and at depths of $4 \mathrm{~mm}$ or more were selected since the main objective of the BF material is to have adequate cure up to 4 or $5 \mathrm{~mm}$ depth as claimed by the manufacturers. The outcome values that were considered acceptable were indicated. For outcomes which were assessed in 2 studies or more, a meta-analyses was performed. A random effects model was used, as we believed that the estimates were not homogenous, and this was confirmed by the $I^{2}$ heterogeneity test, which indicated significant heterogeneity in the summary estimates [29]. The meta-analyses were conducted using Stata 12.2 (StataCorp LP, College Station, TX, USA).

\section{Results}

\subsection{Study Selection}

As presented in Figure 1, a total of 450 studies were identified from the search: 344 from PubMed, 106 from the Cochrane Library. From the titles screening, all studies were relevant to our aim and no studies were excluded. From the abstract screening, 307 studies were excluded for investigating different parameters than the DoC and DC. Of the remining 143 studies which were full text screened, 122 studies were excluded; as 118 
have investigated different parameters than the DoC and DC, two reported in non-English language, and two were systematic reviews. Thus, 21 studies which fulfilled the inclusion criteria of our systematic review, were included for final analysis.

\subsection{Study Characteristics}

Table 1 shows study characteristics of the eligible investigations. All included studies were published between 2013 and 2018, six studies were conducted by multinational collaboration from different countries [30-35]. The sample size of the included studies ranged between three to ten specimens in each study group. The most common type of resin composite reported was Surefil SDR in 17 studies [30-34,36-47]. The studies used four different methods to measure DoC/DC; the most common method was the FTIR method reported in eight studies $[31,32,35,36,41,42,47,48]$ followed by the SM which was reported in five studies $[34,37,38,40,41]$ followed by the Vickers $[36,39,42,49]$ and Knoop [30,33,40,45] Microhardness tests and the RS [43,44,46,50] which were each reported in four different studies. Four studies used multiple measuring methods [36,40-42], while the other 17 studies used a single method each.

\subsection{Results of the Individual Studies}

Twenty one studies investigated the efficiency of polymerization of BFCs reporting seven BFCs and nine BFFs. Characteristics of the different composite materials are presented in Table 2 including the type of resin matrix, main fillers type, filler load, photo initiator type, and manufacturer's details.

The DC estimates from studies using FTIR and RS are presented in Table 3. The highest reported DC value by the FTIR method was for Venus Bulk-Fill (86.07\%) [48] and the lowest reported value was for Tetric N-Ceram Bulk-Fill (24.12\%) [47]. The highest reported DC value by the RS was for Surefil SDR (79.0\%) [44], and the lowest reported value was for Filtek Bulk-Fill (19.41\%) [46].

Table 4 illustrates the remaining thickness values of the studies that used the SM to asses DoC. It also presented the top and bottom microhardness values, and the bottom-totop MHRs. The highest reported remaining thickness value was for X-tra base $(5.68 \mathrm{~mm})$ by Benetti, 2015 [37], and the lowest reported remaining thickness value was for Beautifil Bulk Restorative (2.9 $\mathrm{mm}$ ) by Yap, 2016 [45]. The highest reported MHR was for Venus Bulk-Fill (1.14) by Czasch, 2013 [36]; and the lowest reported MHR was for SonicFill (0.1) by Alshaafi, 2016 [33].

\subsection{Risk of Bias}

None of the studies showed a significant risk of bias, thus none were excluded from the meta-analyses.

\subsection{Synthesis of Results}

Table 5 presents the DC of the studies which used the RS and FTIR methods, but only for the fixed parameters set $(20 \mathrm{~s}$ light-curing time-at the top, 4 and $6 \mathrm{~mm}$ depths, and at 0 and $24 \mathrm{~h}$ times as well as irradiance values). For the DC values at $0 \mathrm{~h}$ : on the top ranged between $41.9 \%$ and $71.46 \%$ and at $4 \mathrm{~mm}$ depth between $24.12 \%$ and $78.8 \%$. At $6 \mathrm{~mm}$, the DC ranged between $36.3 \%$ and $65.6 \%$. For the DC values at $24 \mathrm{~h}$ : on the top ranged between $46.45 \%$ and $74.8 \%$ and at $4 \mathrm{~mm}$ between $32.71 \%$ and $80.07 \%$.

Table 6 illustrates the remaining thickness values for the SM and the MHR values for the fixed parameters set (20 s light-curing time-and 3, 4, 5, and $6 \mathrm{~mm}$ depth, as well as irradiance values). The remaining thickness values for the SM ranged between 2.9 and 5.68 $\mathrm{mm}$. The MHR values: at $3 \mathrm{~mm}$ ranged between 0.68 and 0.98 , at $4 \mathrm{~mm}$ between 0.47 and 1.12 , at $5 \mathrm{~mm}$ between 0.21 and 0.62 , and at $6 \mathrm{~mm}$ between 0.1 and 1.04 . 
Table 1. Summary of the eligible studies characteristics.

\begin{tabular}{|c|c|c|c|c|c|c|c|c|c|c|c|c|c|c|c|c|c|c|c|c|c|c|}
\hline \multirow[b]{2}{*}{ Study } & \multirow[b]{2}{*}{ Country } & \multirow[b]{2}{*}{ Sample Size } & \multicolumn{7}{|c|}{ Bulk-Fills } & \multicolumn{9}{|c|}{ Bulk-Fill Flowables } & \multicolumn{4}{|c|}{ Analysis Methods } \\
\hline & & & 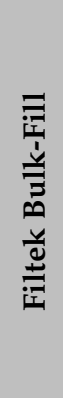 & 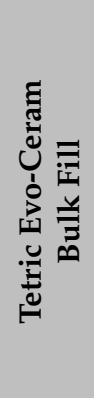 & 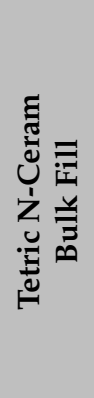 & 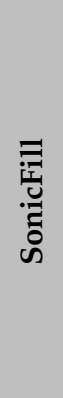 & 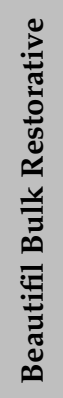 & 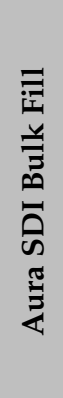 & 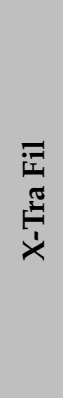 & 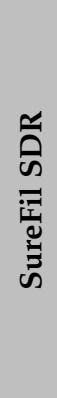 & 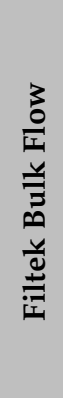 & 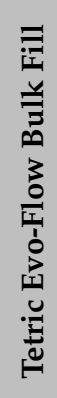 & 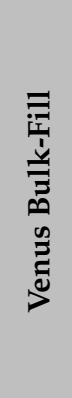 & 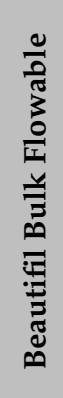 & 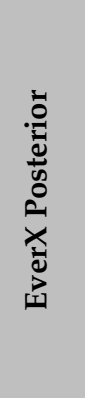 & 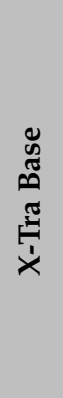 & $\stackrel{\vec{I}}{\Sigma}$ & 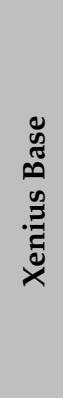 & 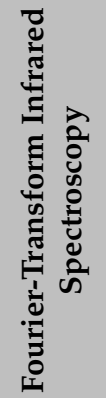 & 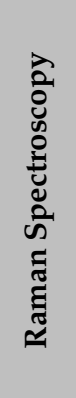 & 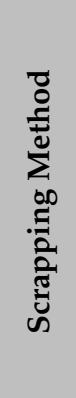 & 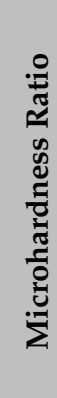 \\
\hline Czasch, 2013 & Germany & 6 & & & & & & & & $\checkmark$ & & & $\checkmark$ & & & & & & $\checkmark$ & & & $\checkmark$ \\
\hline Benetti, 2013 & Denmark & 3 & & $\checkmark$ & & $\checkmark$ & & & & $\checkmark$ & & & $\checkmark$ & & & $\checkmark$ & & & & & $\checkmark$ & \\
\hline Garoushi, 2013 & Finland & 5 & $\checkmark$ & $\checkmark$ & & $\checkmark$ & & & & $\checkmark$ & & & $\checkmark$ & & & $\checkmark$ & & $\checkmark$ & & & $\checkmark$ & \\
\hline El-Damanhoury, 2014 & United States * & 5 & & $\checkmark$ & & & & & $\checkmark$ & $\checkmark$ & & & $\checkmark$ & & & & & & & & & $\checkmark$ \\
\hline Garcia, 2014 & United States & 10 & & & & $\checkmark$ & & & & $\checkmark$ & & & $\checkmark$ & & & & & & & & $\checkmark$ & $\checkmark$ \\
\hline Goracci, 2014 & Italy & 5 & & & & $\checkmark$ & & & & $\checkmark$ & & & & & $\checkmark$ & & & & $\checkmark$ & & $\checkmark$ & \\
\hline Jang, 2015 & South Korea & 8 & & & $\checkmark$ & & & & & $\checkmark$ & & & $\checkmark$ & & & & & & & & & $\checkmark$ \\
\hline Marovic, 2015 & Croatia * & 5 & & & & & & & & $\checkmark$ & & & $\checkmark$ & & & $\checkmark$ & & & $\checkmark$ & & & \\
\hline Li, 2015 & Belgium & 9 & & $\checkmark$ & & & & & & $\checkmark$ & $\checkmark$ & & & & $\checkmark$ & & & & & $\checkmark$ & & \\
\hline Ilie, 2015 & Germany* & 6 & & $\checkmark$ & & & $\checkmark$ & & & $\checkmark$ & & & & $\checkmark$ & & & & & $\checkmark$ & & & \\
\hline Pongprueksa, 2015 & Belgium & 10 & & & & & & & & & $\checkmark$ & & & & & & & & & $\checkmark$ & & \\
\hline Nagi, 2015 & Egypt & 5 & & $\checkmark$ & & & & & $\checkmark$ & & & & & & & & & & & & & $\checkmark$ \\
\hline Al-Ahdal, 2015 & Germany* & 6 & $\checkmark$ & $\checkmark$ & & $\checkmark$ & $\checkmark$ & & & & & & $\checkmark$ & $\checkmark$ & $\checkmark$ & $\checkmark$ & & & $\checkmark$ & & & \\
\hline Alshaafi, 2016 & Canada * & 5 & & $\checkmark$ & & $\checkmark$ & & & $\checkmark$ & $\checkmark$ & & & & & & & & & & & & $\checkmark$ \\
\hline Par, 2016 & Croatia & 4 & & & & & & & & $\checkmark$ & & $\checkmark$ & & & & & & & & $\checkmark$ & & \\
\hline Yap, 2016 & Singapore & 5 & & & $\checkmark$ & & $\checkmark$ & & & $\checkmark$ & & & & $\checkmark$ & $\checkmark$ & & & & & & & $\checkmark$ \\
\hline Lempel, 2016 & Hungary & 5 & $\checkmark$ & & & & & & & $\checkmark$ & & & & & & $\checkmark$ & & & & $\checkmark$ & & \\
\hline Tsujimoto, 2017 & Japan * & 10 & $\checkmark$ & $\checkmark$ & & & $\checkmark$ & & & $\checkmark$ & $\checkmark$ & & & $\checkmark$ & & & & & & & $\checkmark$ & \\
\hline Gonçalve, 2018 & Brazil & 5 & $\checkmark$ & & & $\checkmark$ & & $\checkmark$ & & & $\checkmark$ & & $\checkmark$ & & $\checkmark$ & & & & $\checkmark$ & & & \\
\hline Jain, 2018 & India & 5 & & & $\checkmark$ & & & & & $\checkmark$ & & & & $\checkmark$ & & & $\checkmark$ & & $\checkmark$ & & & \\
\hline
\end{tabular}

${ }^{*}$ Multinational studies; country of the corresponding author is presented. 
Table 2. Summary of bulk-fill resin composite materials found in the cited publications.

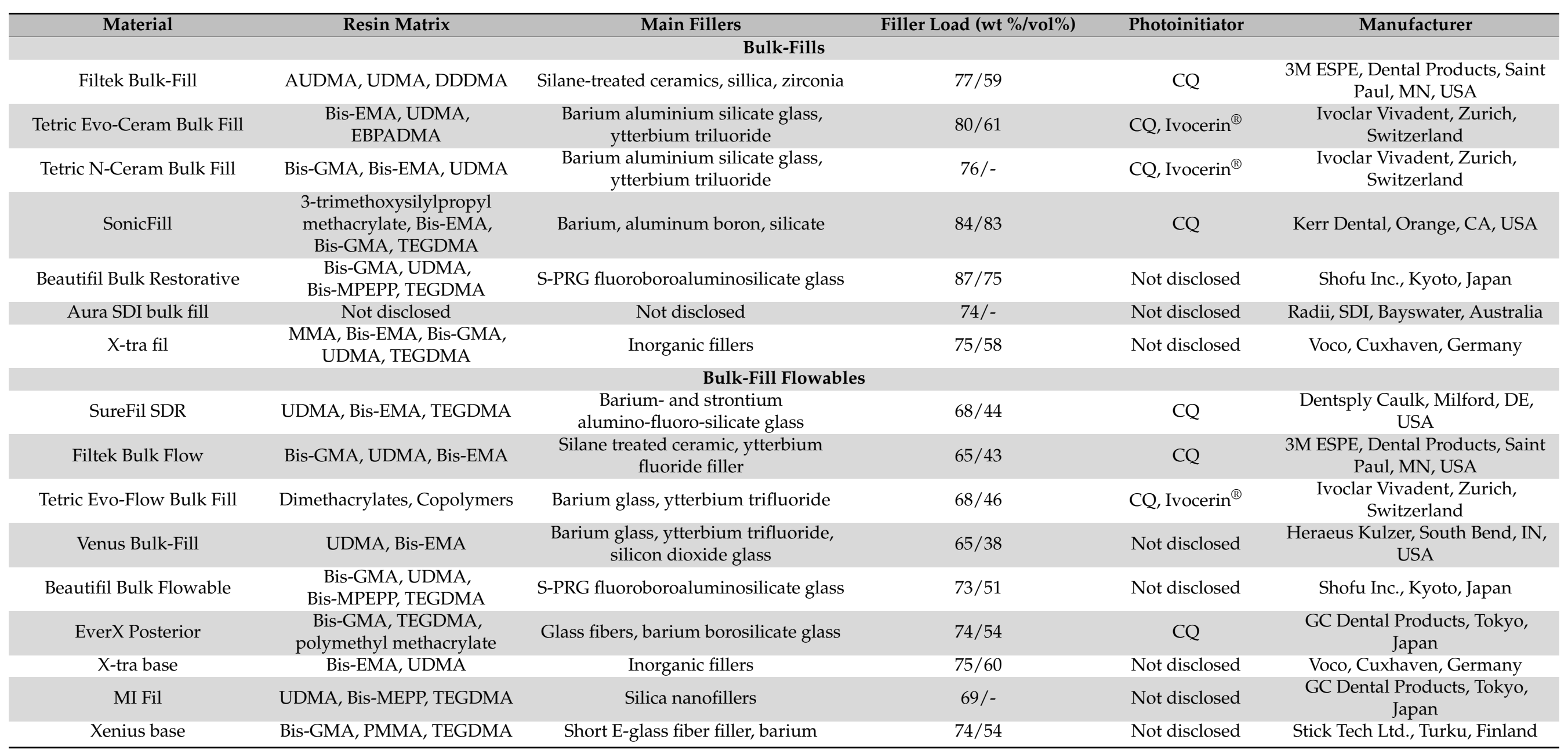

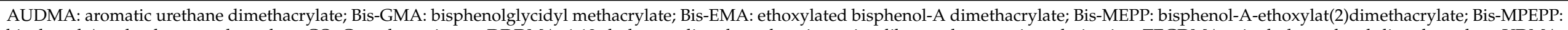

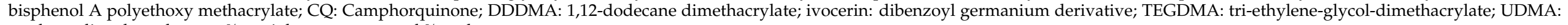

urethane dimethacrylate; $\mathrm{wt} \%$ : weight percentage; vol.\%: volume percentage. 
Table 3. Means and standard deviations (in parenthesis) of degree of conversion data depicted from direct methods.

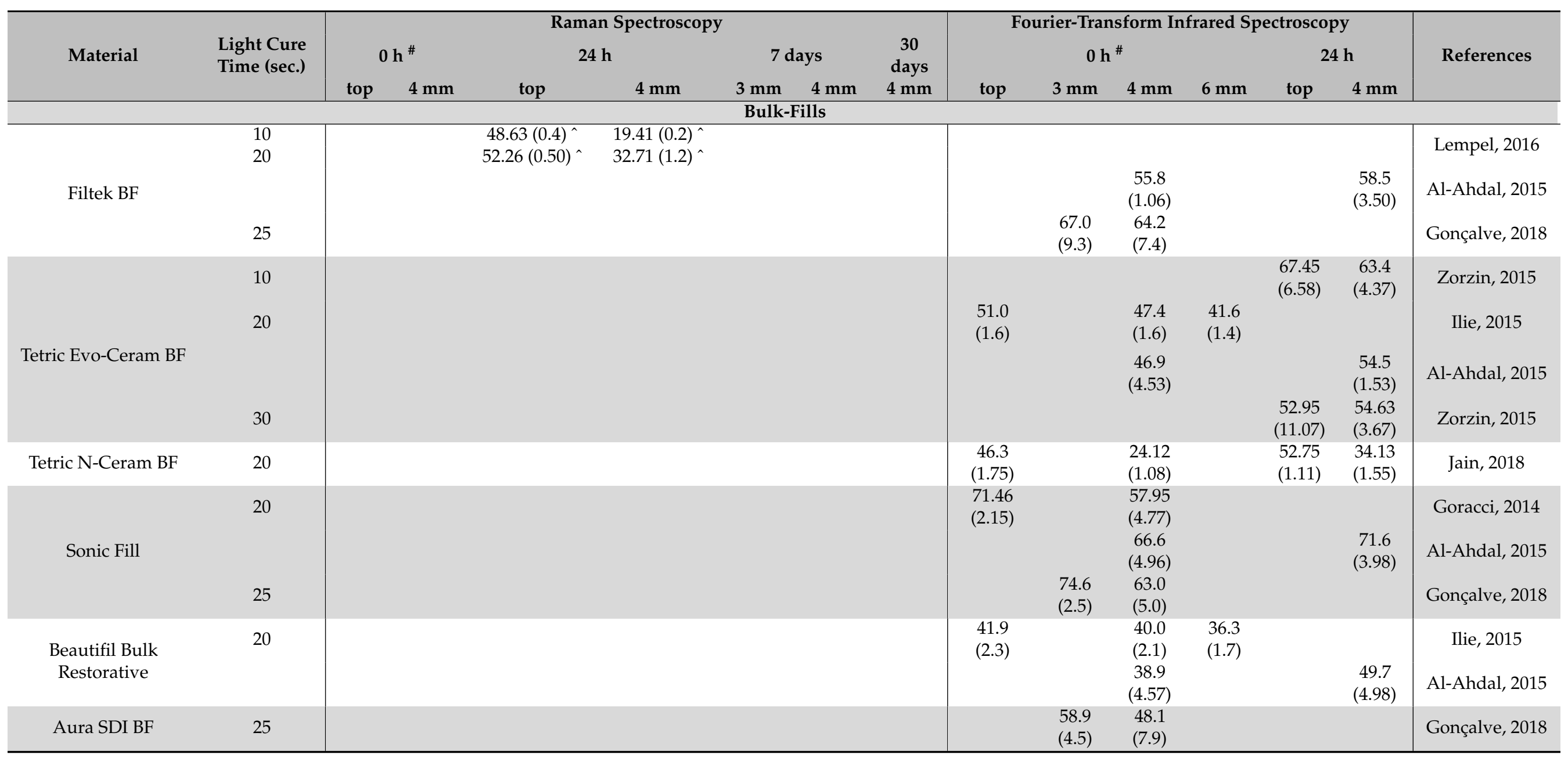


Table 3. Cont

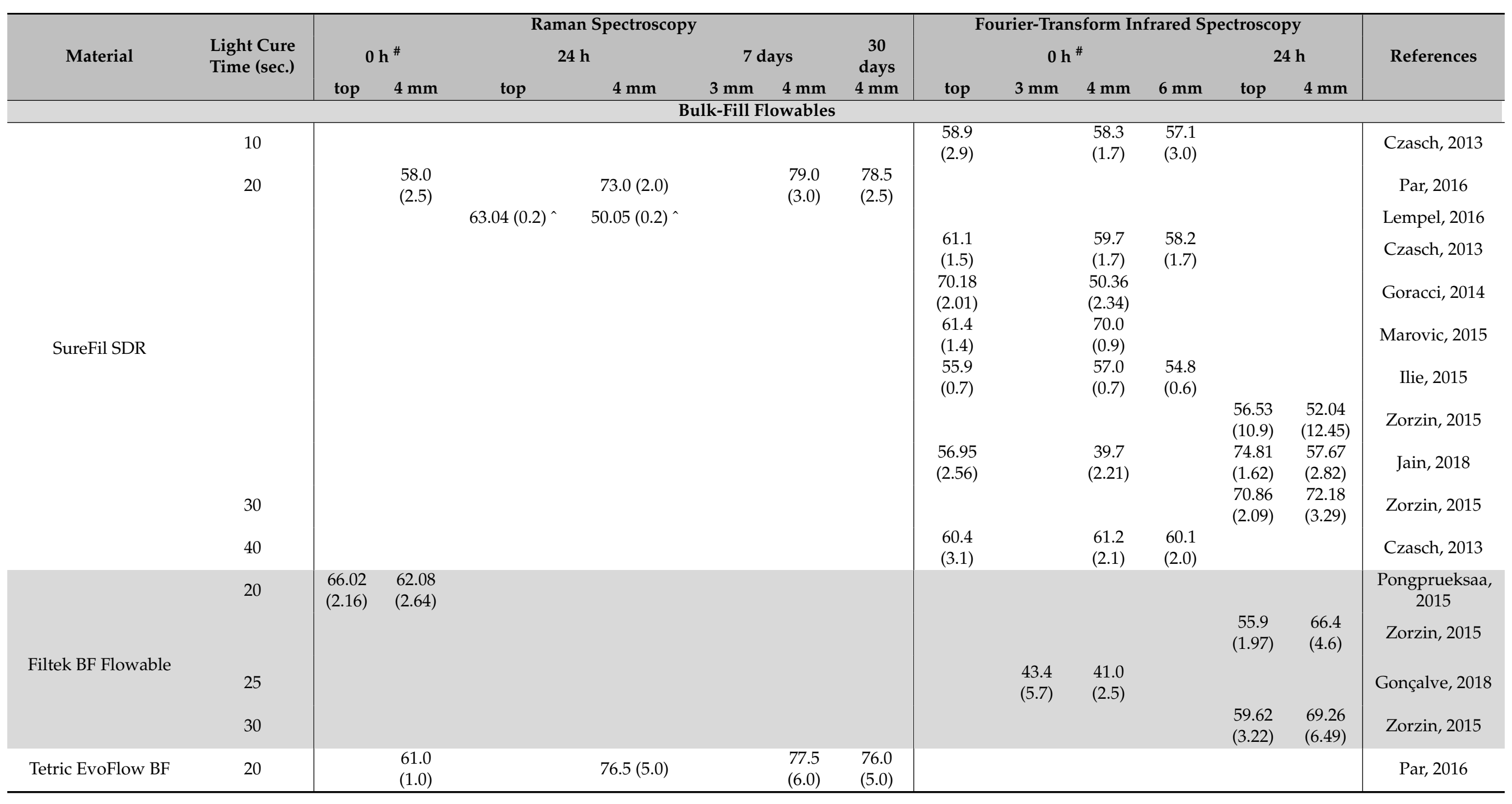


Table 3. Cont.

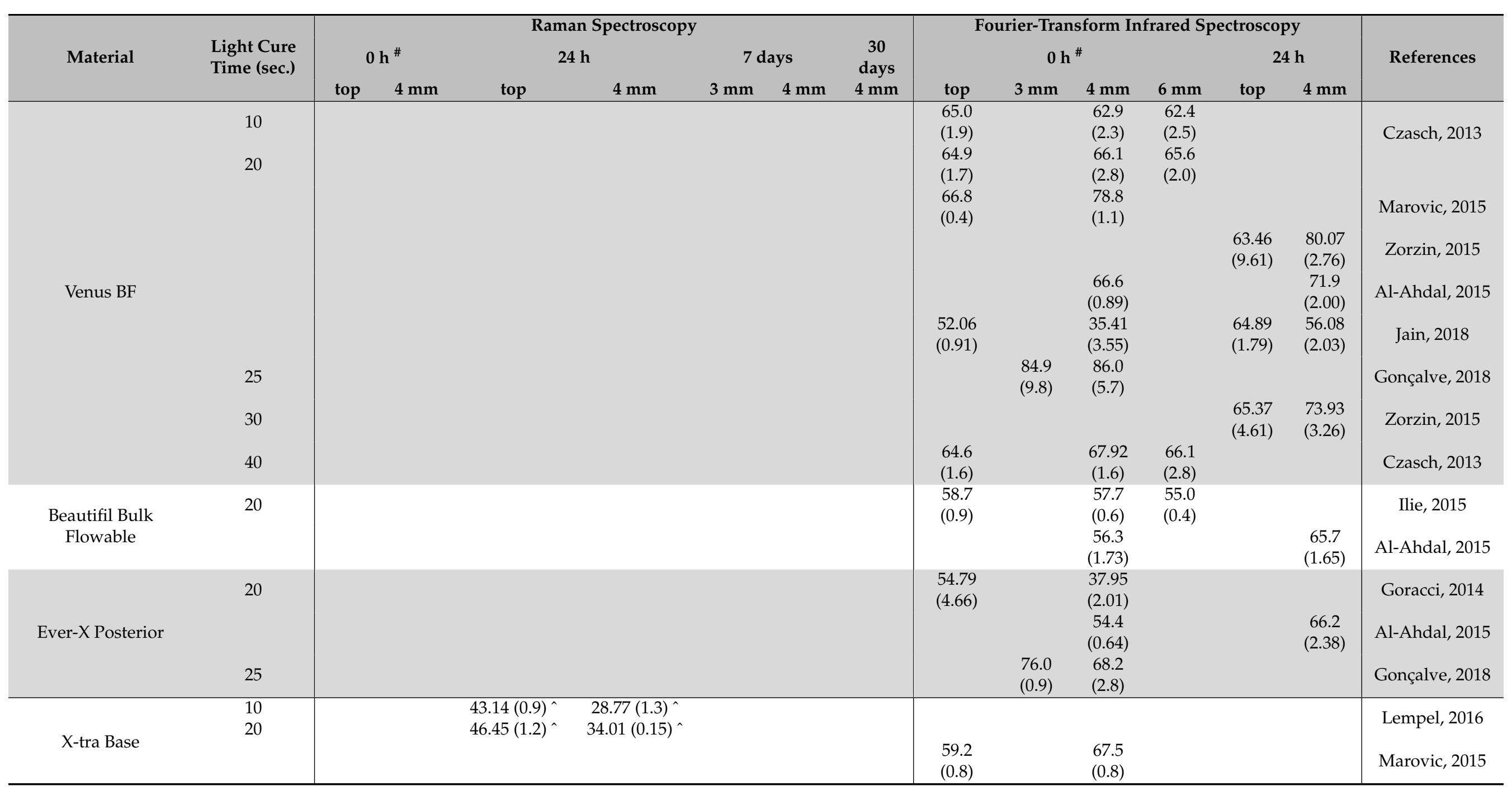


Table 3. Cont.

\begin{tabular}{|c|c|c|c|c|c|c|c|c|c|c|c|c|c|c|c|}
\hline \multirow{3}{*}{ Material } & \multirow{3}{*}{$\begin{array}{l}\text { Light Cure } \\
\text { Time (sec.) }\end{array}$} & \multicolumn{7}{|c|}{ Raman Spectroscopy } & \multicolumn{6}{|c|}{ Fourier-Transform Infrared Spectroscopy } & \multirow{3}{*}{ References } \\
\hline & & \multicolumn{2}{|c|}{$0 \mathrm{~h}^{\#}$} & \multicolumn{2}{|c|}{$24 \mathrm{~h}$} & \multicolumn{2}{|c|}{7 days } & \multirow{2}{*}{$\begin{array}{c}30 \\
\text { days } \\
4 \mathrm{~mm}\end{array}$} & \multicolumn{4}{|c|}{$\mathbf{O} \mathrm{h}^{\#}$} & \multicolumn{2}{|c|}{$24 \mathrm{~h}$} & \\
\hline & & top & $4 \mathrm{~mm}$ & top & $4 \mathrm{~mm}$ & $3 \mathrm{~mm}$ & $4 \mathrm{~mm}$ & & top & $3 \mathrm{~mm}$ & $4 \mathrm{~mm}$ & $6 \mathrm{~mm}$ & top & $4 \mathrm{~mm}$ & \\
\hline & 30 & & & & & & & & & & $\begin{array}{c}49.4 \\
(1.89)\end{array}$ & & $\begin{array}{l}65.24 \\
(2.51) \\
61.48 \\
(3.96)\end{array}$ & $\begin{array}{c}57.7 \\
(3.14) \\
62.53 \\
(3.01) \\
61.53 \\
(4.0)\end{array}$ & $\begin{array}{c}\text { Al-Ahdal, } 2015 \\
\text { Zorzin, } 2015\end{array}$ \\
\hline MI Fil & 20 & & & & & & & & $\begin{array}{l}44.72 \\
(0.86)\end{array}$ & & $\begin{array}{l}24.67 \\
(1.72)\end{array}$ & & $\begin{array}{l}60.78 \\
(2.34)\end{array}$ & $\begin{array}{l}39.7 \\
(1.6)\end{array}$ & Jain, 2018 \\
\hline
\end{tabular}

^ Digitized by WebPlotDigitizer (software details to be added). \# The time at $0 \mathrm{~h}$ corresponds to articles reported values after immediate curing, after $5 \mathrm{~min}$, or after $15 \mathrm{~min}$ postcuring, or unreported time.

Table 4. Means and standard deviations (in parenthesis) of depth of cure data depicted from indirect methods.

\begin{tabular}{|c|c|c|c|c|c|c|c|c|c|c|c|c|c|c|c|}
\hline \multirow{3}{*}{ Material } & \multirow{3}{*}{$\begin{array}{l}\text { Light Cure } \\
\text { Time (sec.) }\end{array}$} & \multirow{3}{*}{$\begin{array}{c}\text { Scrapping } \\
\text { Method } \\
\text { (Remaining } \\
\text { Thickness; } \mathrm{mm} \text { ) }\end{array}$} & \multicolumn{12}{|c|}{ Microhardness } & \multirow{3}{*}{ References } \\
\hline & & & \multicolumn{4}{|c|}{ Top } & \multicolumn{4}{|c|}{ Bottom } & \multicolumn{4}{|c|}{ Ratio } & \\
\hline & & & $3 \mathrm{~mm}$ & $4 \mathrm{~mm}$ & $5 \mathrm{~mm}$ & $6 \mathrm{~mm}$ & $3 \mathrm{~mm}$ & $4 \mathrm{~mm}$ & $5 \mathrm{~mm}$ & $6 \mathrm{~mm}$ & $3 \mathrm{~mm}$ & $4 \mathrm{~mm}$ & $5 \mathrm{~mm}$ & $6 \mathrm{~mm}$ & \\
\hline \multicolumn{16}{|c|}{ Bulk-Fills } \\
\hline Filtek BF & $\begin{array}{l}20 \\
30 \\
40\end{array}$ & $\begin{array}{l}4.64(0.1) \wedge \\
3.57(0.11) \\
3.81(0.09) \\
4.14(0.09)\end{array}$ & & & & & & & & & & & & & $\begin{array}{l}\text { Garoushi, } 2013 \\
\text { Tsujimoto, } 2017\end{array}$ \\
\hline \multirow{3}{*}{$\begin{array}{c}\text { Tetric } \\
\text { Evo-Ceram } \\
\text { BF }\end{array}$} & 10 & & $\begin{array}{c}\mathrm{VHN}= \\
57.00 \\
(1.74)\end{array}$ & $\begin{array}{l}\mathrm{VHN}= \\
58.69 \\
(0.72)\end{array}$ & & & $\begin{array}{c}\mathrm{VHN}= \\
48.12 \\
(3.94)\end{array}$ & $\begin{array}{c}\mathrm{VHN}= \\
51.81 \\
(1.89)\end{array}$ & & & 0.84 * & 0.88 * & & & Nagi, 2015 \\
\hline & & & & $\begin{array}{c}\mathrm{VHN}= \\
64.52 \\
(2.45)\end{array}$ & & & & $\begin{array}{c}\mathrm{VHN}= \\
41.92 \\
(4.46)\end{array}$ & & & & 0.65 & & & Zorzin, 2015 \\
\hline & 20 & $\begin{array}{l}3.86(0.07) \\
3.82(0.08)\end{array}$ & & & & & & & & & & & & & $\begin{array}{c}\text { Tsujimoto, } 2017 \\
\text { Benetti, } 2015\end{array}$ \\
\hline
\end{tabular}


Table 4. Cont.

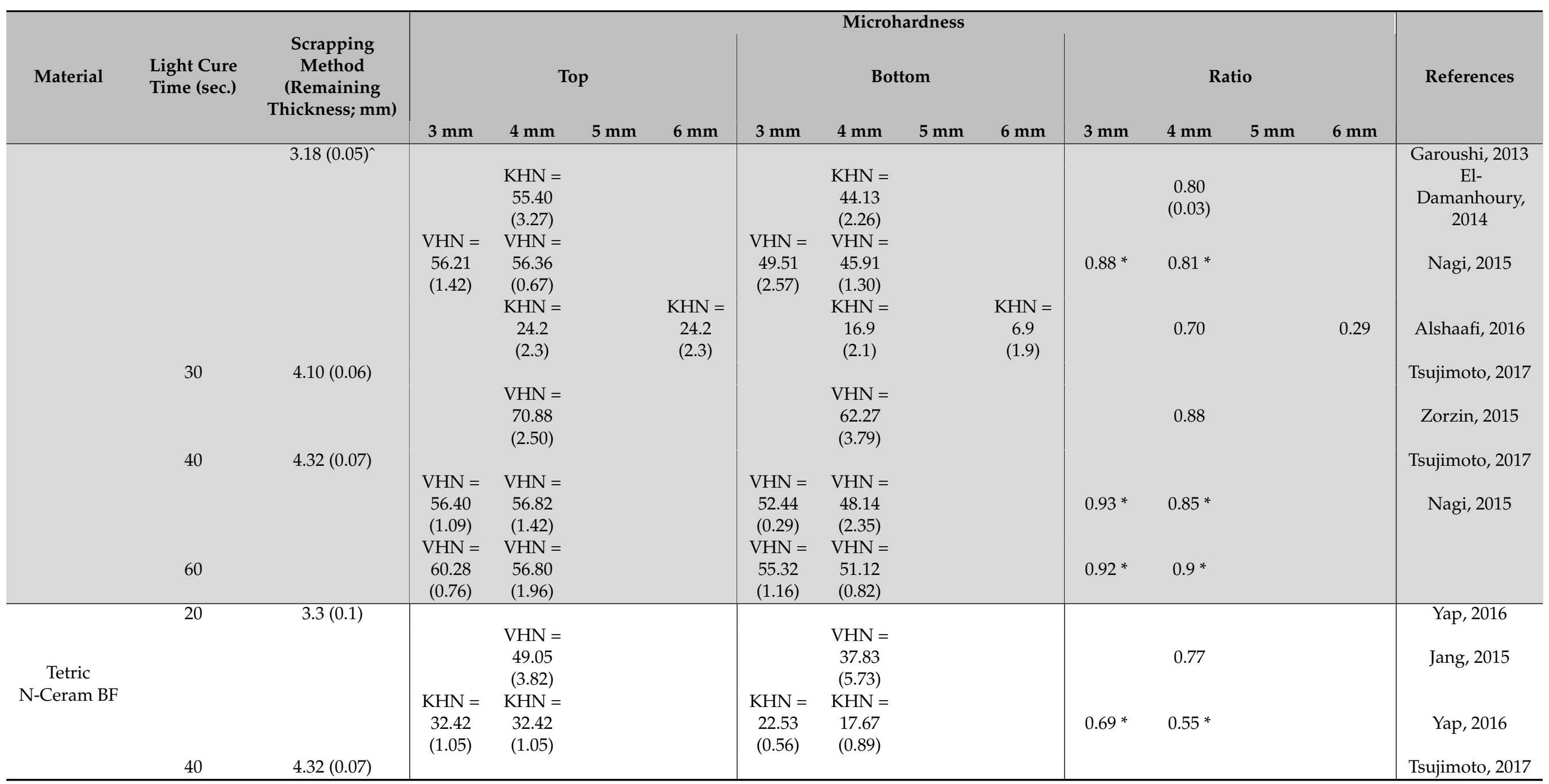


Table 4. Cont.

\begin{tabular}{|c|c|c|c|c|c|c|c|c|c|c|c|c|c|c|c|}
\hline \multirow[t]{2}{*}{ Material } & \multirow[t]{2}{*}{$\begin{array}{l}\text { Light Cure } \\
\text { Time (sec.) }\end{array}$} & \multirow{2}{*}{$\begin{array}{c}\text { Scrapping } \\
\text { Method } \\
\text { (Remaining } \\
\text { Thickness; mm) }\end{array}$} & & & & & & $\begin{array}{r}\text { Microl } \\
\text { Bo }\end{array}$ & ardness & & & & & & \multirow[t]{2}{*}{ References } \\
\hline & & & $3 \mathrm{~mm}$ & $4 \mathrm{~mm}$ & $5 \mathrm{~mm}$ & $6 \mathrm{~mm}$ & $3 \mathrm{~mm}$ & $4 \mathrm{~mm}$ & $5 \mathrm{~mm}$ & $6 \mathrm{~mm}$ & $3 \mathrm{~mm}$ & $4 \mathrm{~mm}$ & $5 \mathrm{~mm}$ & $6 \mathrm{~mm}$ & \\
\hline Sonic Fill & 20 & $\begin{array}{l}3.20(0.1)^{\wedge} \\
3.46(0.08) \\
3.47(0.03) \\
3.43(0.07)\end{array}$ & $\begin{array}{c}\mathrm{KHN}= \\
72.3 \\
(3.20)\end{array}$ & $\begin{array}{c}\mathrm{KHN}= \\
72.4 \\
(2.11) \\
\\
\mathrm{KHN}= \\
35.7 \\
(2.4)\end{array}$ & $\begin{array}{c}\mathrm{KHN}= \\
71.1 \\
(2.64)\end{array}$ & $\begin{array}{c}\mathrm{KHN}= \\
35.7 \\
(2.4)\end{array}$ & $\begin{array}{c}\mathrm{KHN}= \\
48.9 \\
(2.06)\end{array}$ & $\begin{array}{c}\mathrm{KHN}= \\
34.0 \\
(1.88) \\
\\
\mathrm{KHN}= \\
24.9 \\
(2.5)\end{array}$ & $\begin{array}{c}\mathrm{KHN}= \\
15.1 \\
(1.29)\end{array}$ & $\begin{array}{c}\mathrm{KHN}= \\
3.4 \\
(1.3)\end{array}$ & $\begin{array}{c}0.68 \\
(0.05)\end{array}$ & $\begin{array}{c}0.47 \\
(0.04)\end{array}$ & $\begin{array}{c}0.21 \\
(0.02)\end{array}$ & 0.10 & $\begin{array}{l}\text { Garoushi,2013 } \\
\text { Garcia, } 2014 \\
\text { Goracci, } 2014 \\
\text { Benetti, } 2015 \\
\text { Alshaafi, } 2016\end{array}$ \\
\hline $\begin{array}{l}\text { Beautifil } \\
\text { Bulk } \\
\text { Restorative }\end{array}$ & $\begin{array}{l}20 \\
\\
30 \\
40\end{array}$ & $\begin{array}{c}2.9(0.1) \\
3.42(0.09) \\
3.72(0.08) \\
4.02(0.08)\end{array}$ & $\begin{array}{c}\mathrm{KHN}= \\
34.58 \\
(0.69)\end{array}$ & & & & $\begin{array}{c}\mathrm{KHN}= \\
27.15 \\
(1.1)\end{array}$ & & & & 0.79 * & - & - & & $\begin{array}{c}\text { Yap, } 2016 \\
\text { Tsujimoto, } 2017\end{array}$ \\
\hline X-tra Fil & $\begin{array}{l}10 \\
20\end{array}$ & & $\begin{array}{c}\mathrm{VHN}= \\
93.76 \\
(1.89) \\
\\
\\
\mathrm{VHN}= \\
95.39 \\
(0.37)\end{array}$ & $\begin{array}{c}\mathrm{VHN}= \\
93.76 \\
(1.89) \\
\mathrm{KHN}= \\
59.07 \\
(6.37) \\
\mathrm{VHN}= \\
95.90 \\
(0.45) \\
\mathrm{KHN}= \\
36.2 \\
(2.9)\end{array}$ & & $\begin{array}{c} \\
\mathrm{KHN}= \\
36.2 \\
(2.9)\end{array}$ & $\begin{array}{c}\mathrm{VHN}= \\
89.89 \\
(2.40) \\
\\
\\
\mathrm{VHN}= \\
93.87 \\
(0.71)\end{array}$ & $\begin{array}{c}\mathrm{VHN}= \\
89.89 \\
(2.40) \\
\mathrm{KHN}= \\
51.20 \\
(7.61) \\
\mathrm{VHN}= \\
94.12 \\
(0.78) \\
\mathrm{KHN}= \\
31.8 \\
(2.8)\end{array}$ & & $\begin{array}{c}\mathrm{KHN}= \\
22.0 \\
(2.1)\end{array}$ & $0.96^{*}$ & $\begin{array}{c}0.96 \text { * } \\
0.86 \\
(0.05) \\
0.98 \text { * } \\
0.88\end{array}$ & & 0.61 & $\begin{array}{c}\text { Nagi, } 2015 \\
\text { El- } \\
\begin{array}{c}\text { Damanhoury, } \\
2014\end{array} \\
\text { Nagi, } 2015 \\
\text { Alshaafi,2016 }\end{array}$ \\
\hline
\end{tabular}


Table 4. Cont.

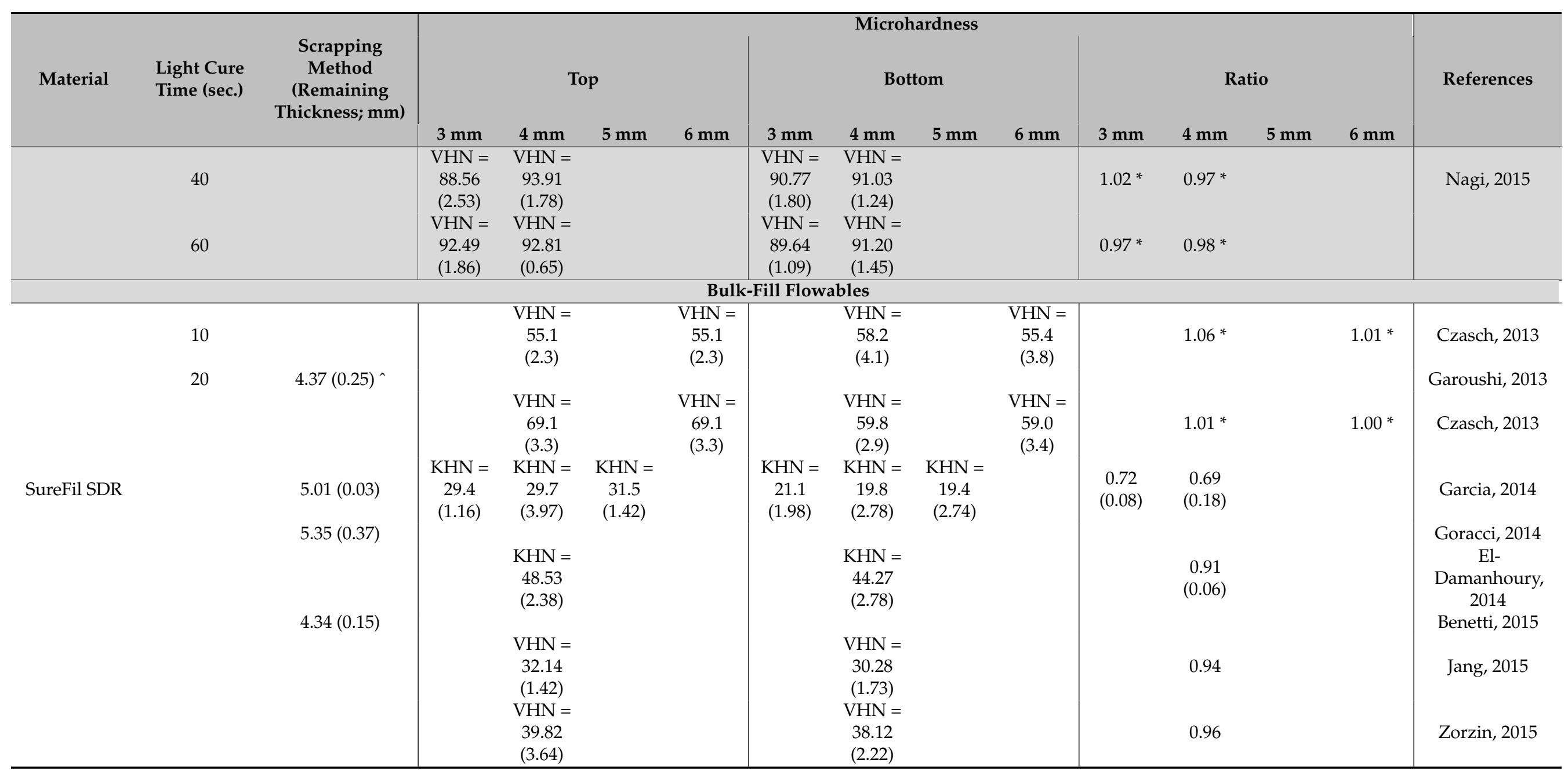


Table 4. Cont.

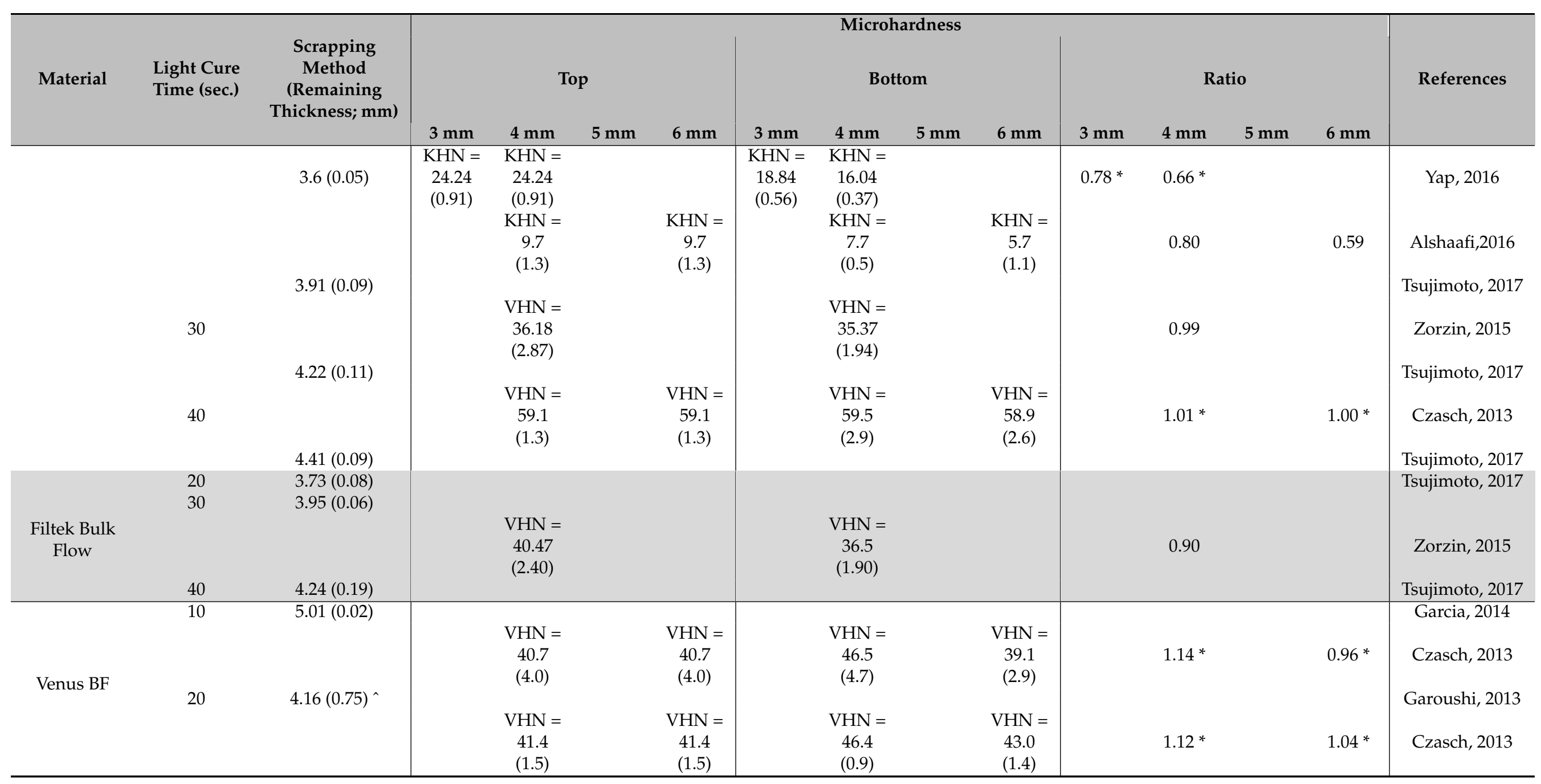


Table 4. Cont.

\begin{tabular}{|c|c|c|c|c|c|c|c|c|c|c|c|c|c|c|c|}
\hline \multirow[t]{2}{*}{ Material } & \multirow[t]{2}{*}{$\begin{array}{l}\text { Light Cure } \\
\text { Time (sec.) }\end{array}$} & \multirow{2}{*}{$\begin{array}{c}\text { Scrapping } \\
\text { Method } \\
\text { (Remaining } \\
\text { Thickness; mm) }\end{array}$} & \multicolumn{4}{|c|}{ Top } & \multicolumn{4}{|c|}{ Microhardness } & \multicolumn{4}{|c|}{ Ratio } & \multirow[t]{2}{*}{ References } \\
\hline & & & $3 \mathrm{~mm}$ & $4 \mathrm{~mm}$ & $5 \mathrm{~mm}$ & $6 \mathrm{~mm}$ & $3 \mathrm{~mm}$ & $4 \mathrm{~mm}$ & $5 \mathrm{~mm}$ & $6 \mathrm{~mm}$ & $3 \mathrm{~mm}$ & $4 \mathrm{~mm}$ & $5 \mathrm{~mm}$ & $6 \mathrm{~mm}$ & \\
\hline & $\begin{array}{l}30 \\
40\end{array}$ & $5.57(0.28)$ & $\begin{array}{c}\mathrm{KHN}= \\
21.3 \\
(1.02)\end{array}$ & $\begin{array}{c}\mathrm{KHN}= \\
23.5 \\
(3.25) \\
\mathrm{KHN}= \\
36.60 \\
(0.97) \\
\\
\mathrm{VHN}= \\
30.55 \\
(1.17) \\
\mathrm{VHN}= \\
34.34 \\
(4.34) \\
\mathrm{VHN}= \\
30.64 \\
(2.12) \\
\mathrm{VHN}= \\
42.7 \\
(4.9)\end{array}$ & $\begin{array}{c}\mathrm{KHN}= \\
23.0 \\
(2.34)\end{array}$ & 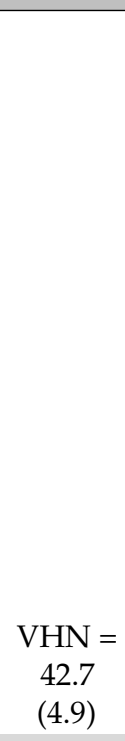 & $\begin{array}{c}\mathrm{KHN}= \\
16.6 \\
(0.70)\end{array}$ & $\begin{array}{c}\mathrm{KHN}= \\
15.6 \\
(2.09) \\
\mathrm{KHN}= \\
35.53 \\
(1.32) \\
\\
\mathrm{VHN}= \\
29.95 \\
(1.16) \\
\mathrm{VHN}= \\
29.15 \\
(1.93) \\
\mathrm{VHN}= \\
34.41 \\
(2.07) \\
\mathrm{VHN}= \\
46.4 \\
(1.0)\end{array}$ & $\begin{array}{c}\mathrm{KHN}= \\
13.5 \\
(0.74)\end{array}$ & 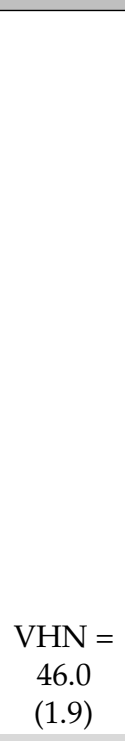 & $\begin{array}{c}0.78 \\
(0.03)\end{array}$ & $\begin{array}{c}0.67 \\
(0.10) \\
\\
0.97 \\
(0.02) \\
\\
0.98 \\
0.85 \\
1.12 \\
1.09 \text { * }\end{array}$ & $\begin{array}{c}0.59 \\
(0.07)\end{array}$ & 1.08 * & 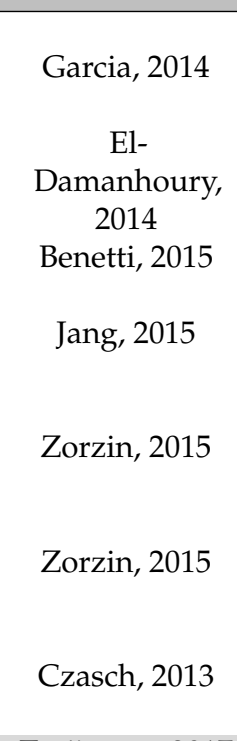 \\
\hline $\begin{array}{l}\text { Beautifil } \\
\text { Bulk } \\
\text { Flowable }\end{array}$ & $\begin{array}{l}30 \\
40\end{array}$ & $\begin{array}{l}3.62(0.06) \\
3.55(0.03) \\
3.86(0.07) \\
4.10(0.07)\end{array}$ & $\begin{array}{c}\mathrm{KHN}= \\
35.74 \\
(1.17)\end{array}$ & $\begin{array}{c}\mathrm{KHN}= \\
35.74 \\
(1.17)\end{array}$ & & & $\begin{array}{c}\mathrm{KHN}= \\
27.16 \\
(0.64)\end{array}$ & $\begin{array}{c}\mathrm{KHN}= \\
18.19 \\
(1.9)\end{array}$ & & & $0.76^{*}$ & 0.51 * & - & & $\begin{array}{c}\text { Tsujimoto, } 2017 \\
\text { Yap, } 2016 \\
\text { Tsujimoto, } 2017\end{array}$ \\
\hline $\begin{array}{c}\text { Ever-X } \\
\text { Posterior }\end{array}$ & 20 & $\begin{array}{l}5.29(0.19) \\
3.6(0.03)\end{array}$ & $\begin{array}{c}\mathrm{KHN}= \\
33.72 \\
(0.89)\end{array}$ & $\begin{array}{c}\mathrm{KHN}= \\
33.72 \\
(0.89)\end{array}$ & $\begin{array}{c}\mathrm{KHN}= \\
33.72 \\
(0.89)\end{array}$ & & $\begin{array}{c}\mathrm{KHN}= \\
24.53 \\
(1.17)\end{array}$ & $\begin{array}{c}\mathrm{KHN}= \\
21.88 \\
(1.07)\end{array}$ & $\begin{array}{c}\mathrm{KHN}= \\
19.43 \\
(0.6)\end{array}$ & & 0.73 * & $0.65 *$ & 0.58 * & & $\begin{array}{c}\text { Goracci, } 2014 \\
\text { Yap, } 2016\end{array}$ \\
\hline
\end{tabular}


Table 4. Cont.

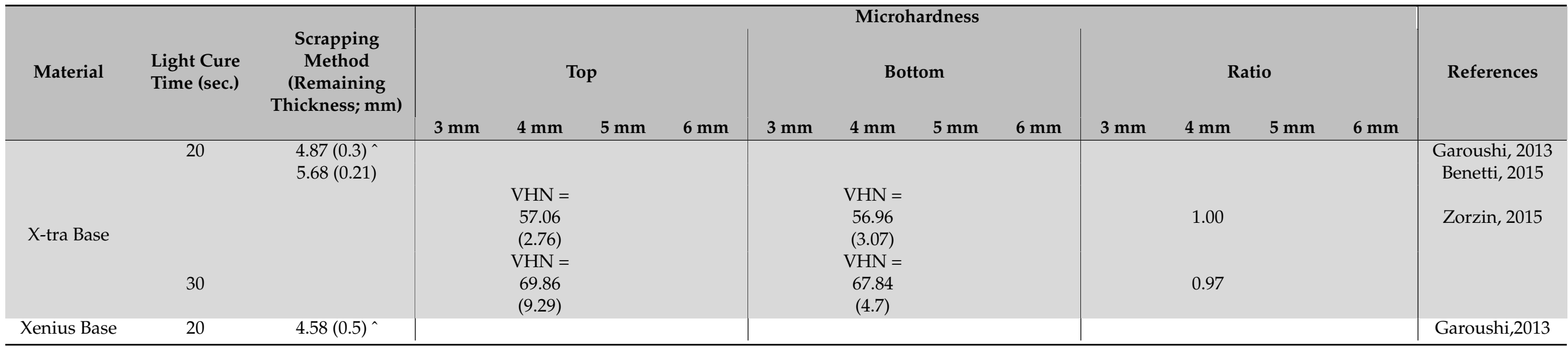

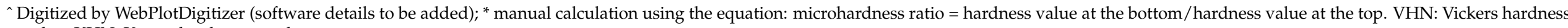
number, KHN: Knoop hardness number.

Table 5. Means and standard deviations (in parenthesis) of degree of conversion data from studies utilizing $20 \mathrm{~s}$ light curing protocol at 4 and $6 \mathrm{~mm}$.

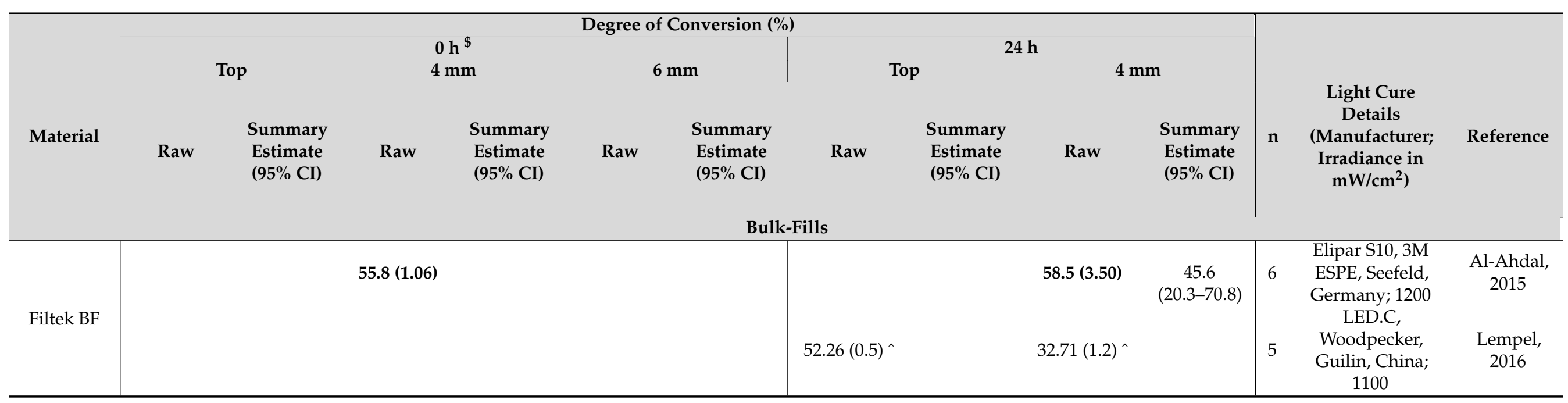


Table 5. Cont.

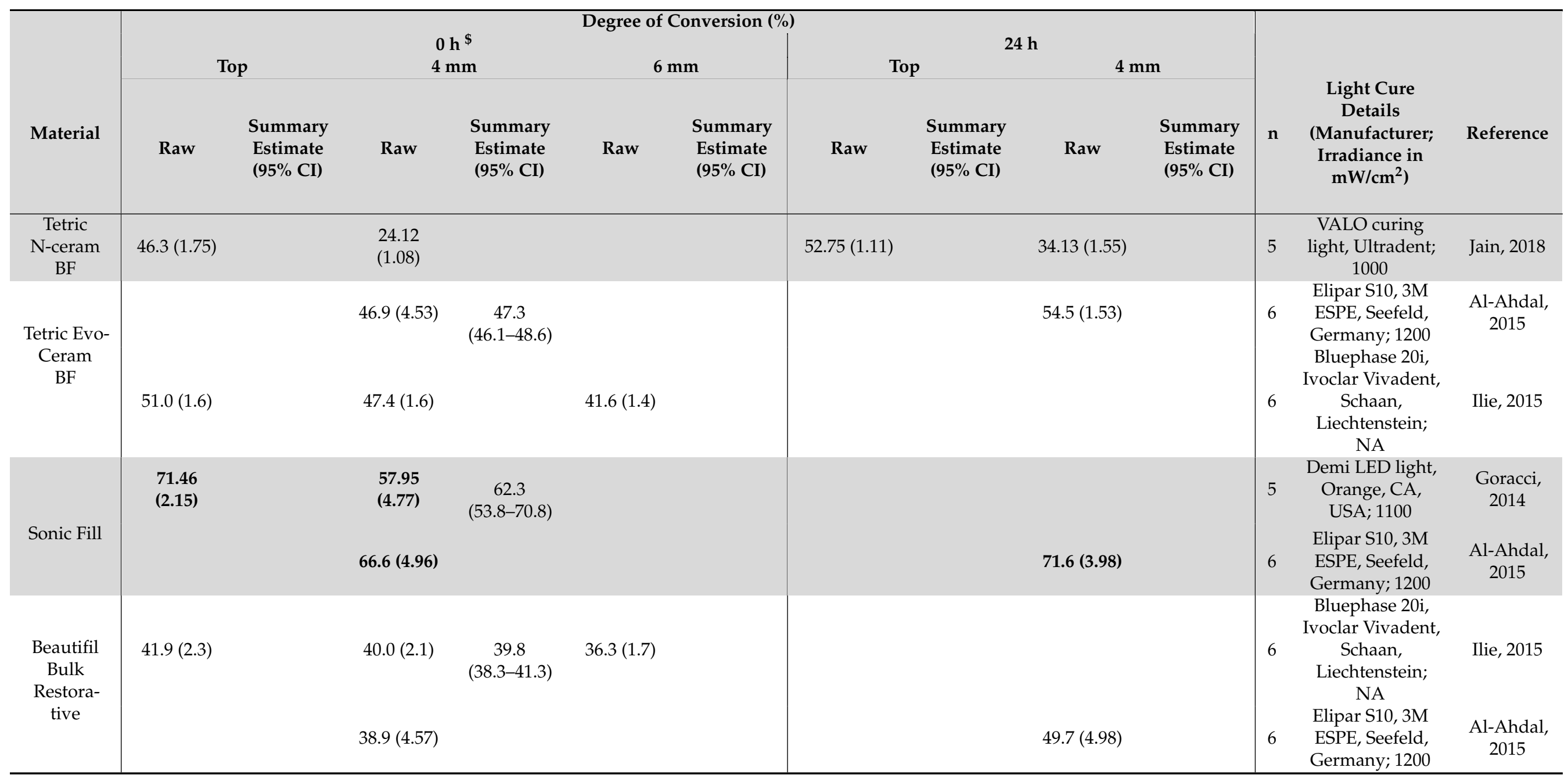


Table 5. Cont.

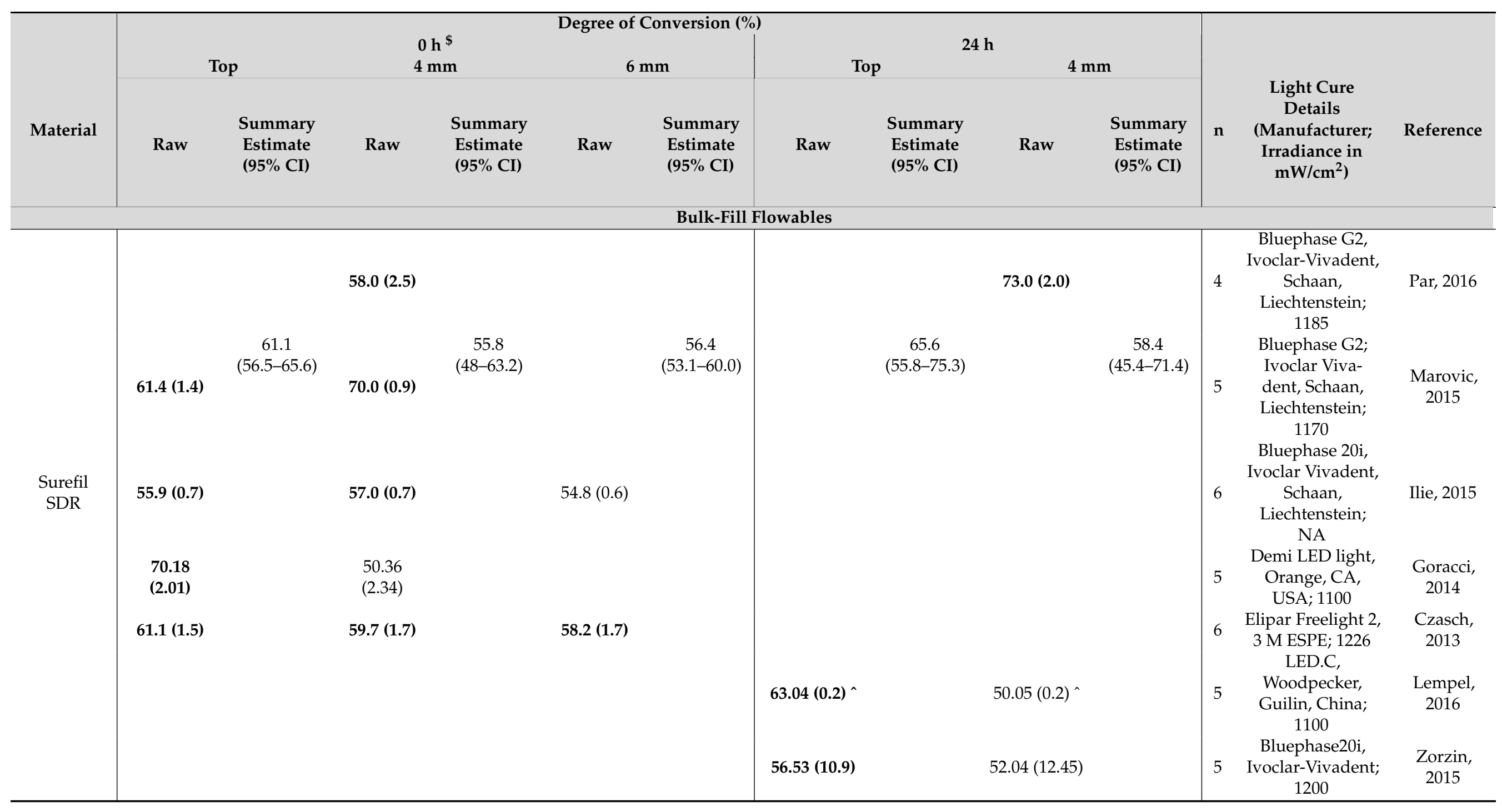


Table 5. Cont.

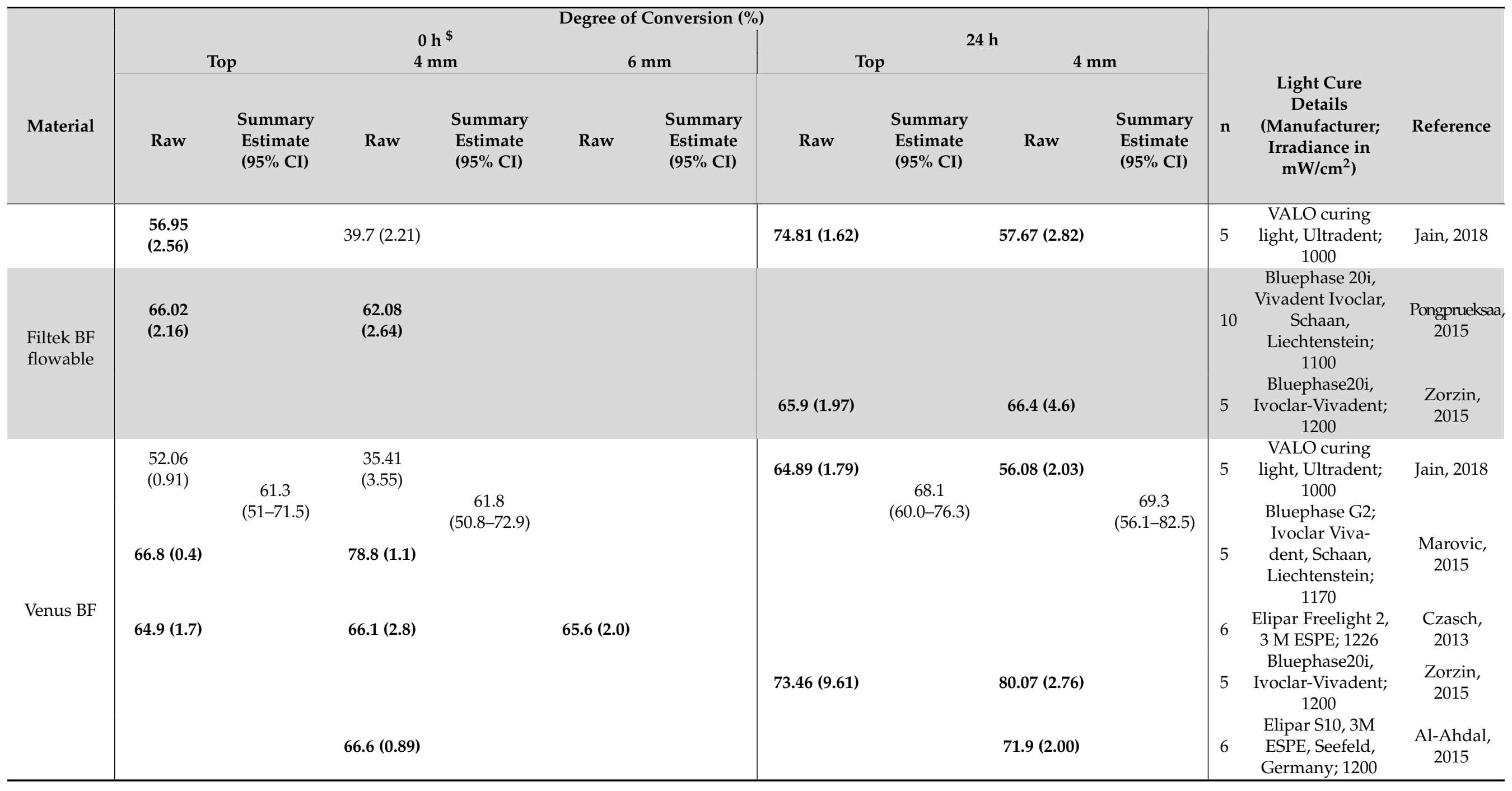


Table 5. Cont.

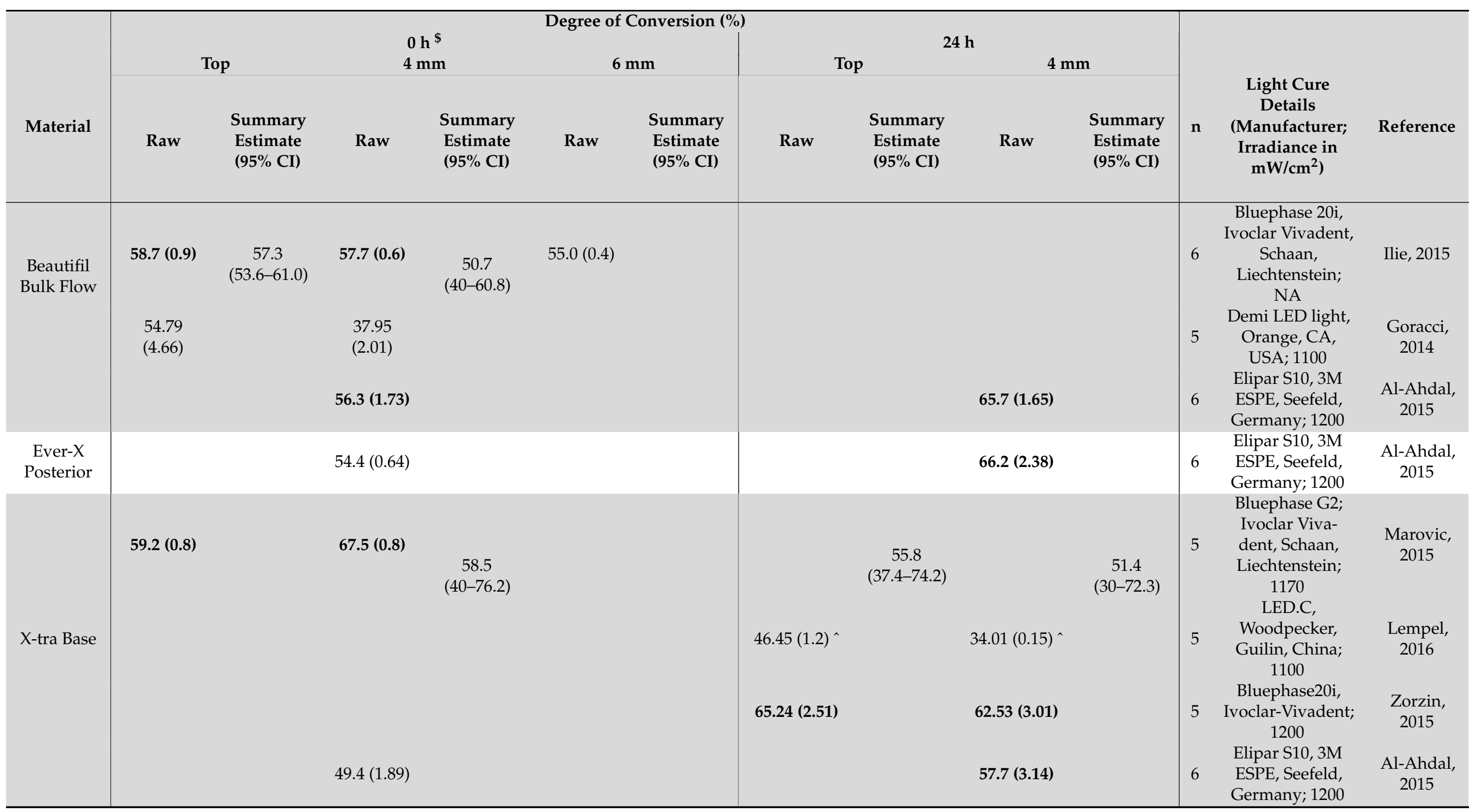


Table 5. Cont.

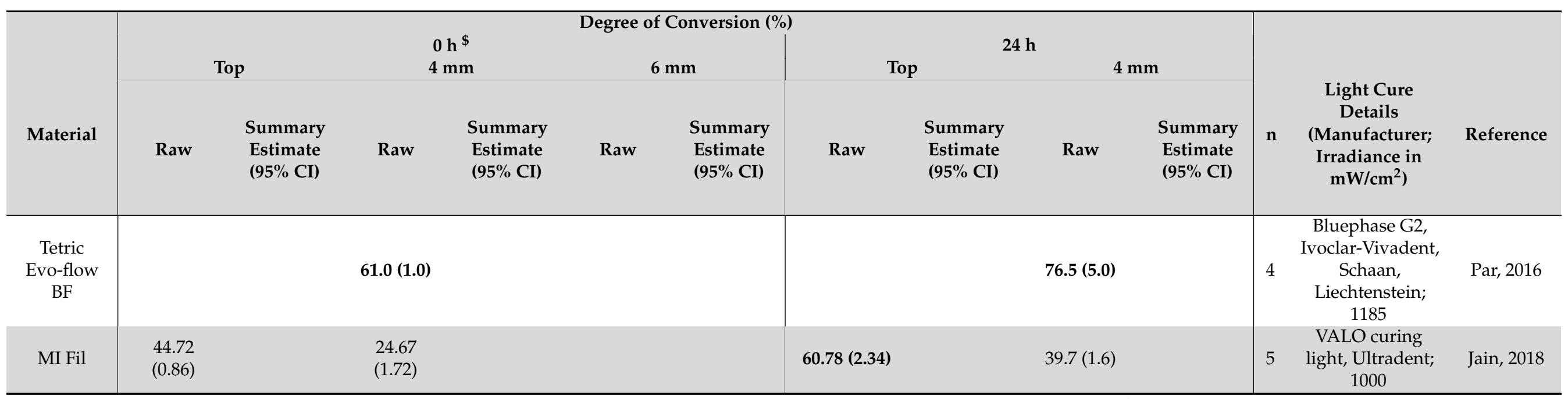

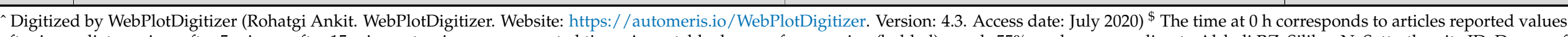

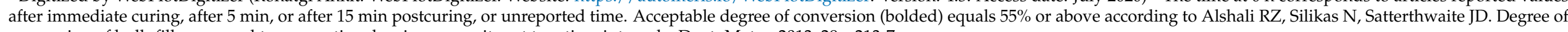
conversion of bulk-fill compared to conventional resin-composites at two time intervals. Dent. Mater. 2013, 29, e213-7.

Table 6. Means and standard deviations (in parenthesis) of depth of cure and microhardness ratio data from studies utilizing $20 \mathrm{~s}$ light curing protocol.

\begin{tabular}{|c|c|c|c|c|c|c|c|c|c|c|c|c|}
\hline \multirow[b]{2}{*}{ Material } & \multicolumn{4}{|c|}{ Scrapping Method } & \multicolumn{7}{|c|}{ Microhardness Ratio } & \multirow[b]{2}{*}{ References } \\
\hline & $\begin{array}{c}\text { Remaining } \\
\text { Thickness } \\
(\mathrm{mm})\end{array}$ & $\mathbf{n}$ & $\begin{array}{l}\text { Light Cure Details } \\
\text { (Manufacturer; } \\
\text { Irradiance in } \mathrm{mW} / \mathrm{cm}^{2} \text { ) }\end{array}$ & $\begin{array}{l}\text { Summary } \\
\text { Estimate } \\
(95 \% \text { CI })\end{array}$ & $3 \mathrm{~mm}$ & Raw & $\begin{array}{c}\text { Summary } \\
\text { Estimate } \\
(95 \% \mathrm{CI})\end{array}$ & $5 \mathrm{~mm}$ & $6 \mathrm{~mm}$ & $\mathbf{n}$ & $\begin{array}{l}\text { Light Cure Details } \\
\text { (Manufacturer; } \\
\text { Irradiance in } \\
\mathrm{mW} / \mathrm{cm}^{2} \text { ) }\end{array}$ & \\
\hline \multicolumn{13}{|c|}{ Bulk-Fills } \\
\hline Filtek BF & $\begin{array}{l}4.64(0.1)^{\wedge} \\
3.57(0.11)\end{array}$ & $\begin{array}{l}3 \\
10\end{array}$ & $\begin{array}{c}\text { TC-01, Spring Health } \\
\text { Products, USA; } 1100 \\
\text { Optilux 501, Kerr, } \\
\text { Orange, CA, USA; } \geq 600\end{array}$ & $4.1(3.1-5.2)$ & & & & & & & & $\begin{array}{l}\text { Garoushi, } \\
2013 \\
\text { Tsujimoto, } \\
2017\end{array}$ \\
\hline
\end{tabular}


Table 6. Cont.

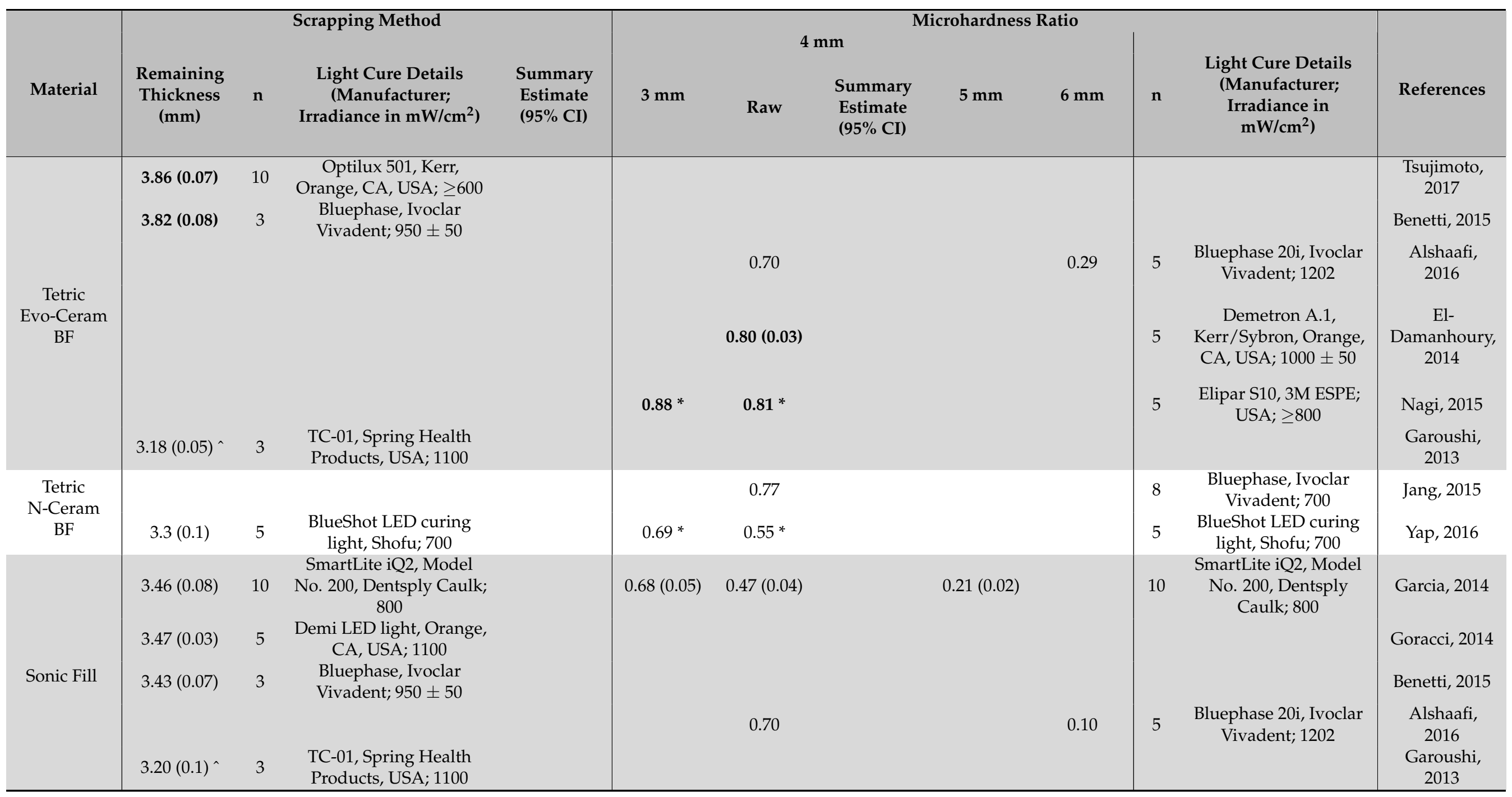


Table 6. Cont.

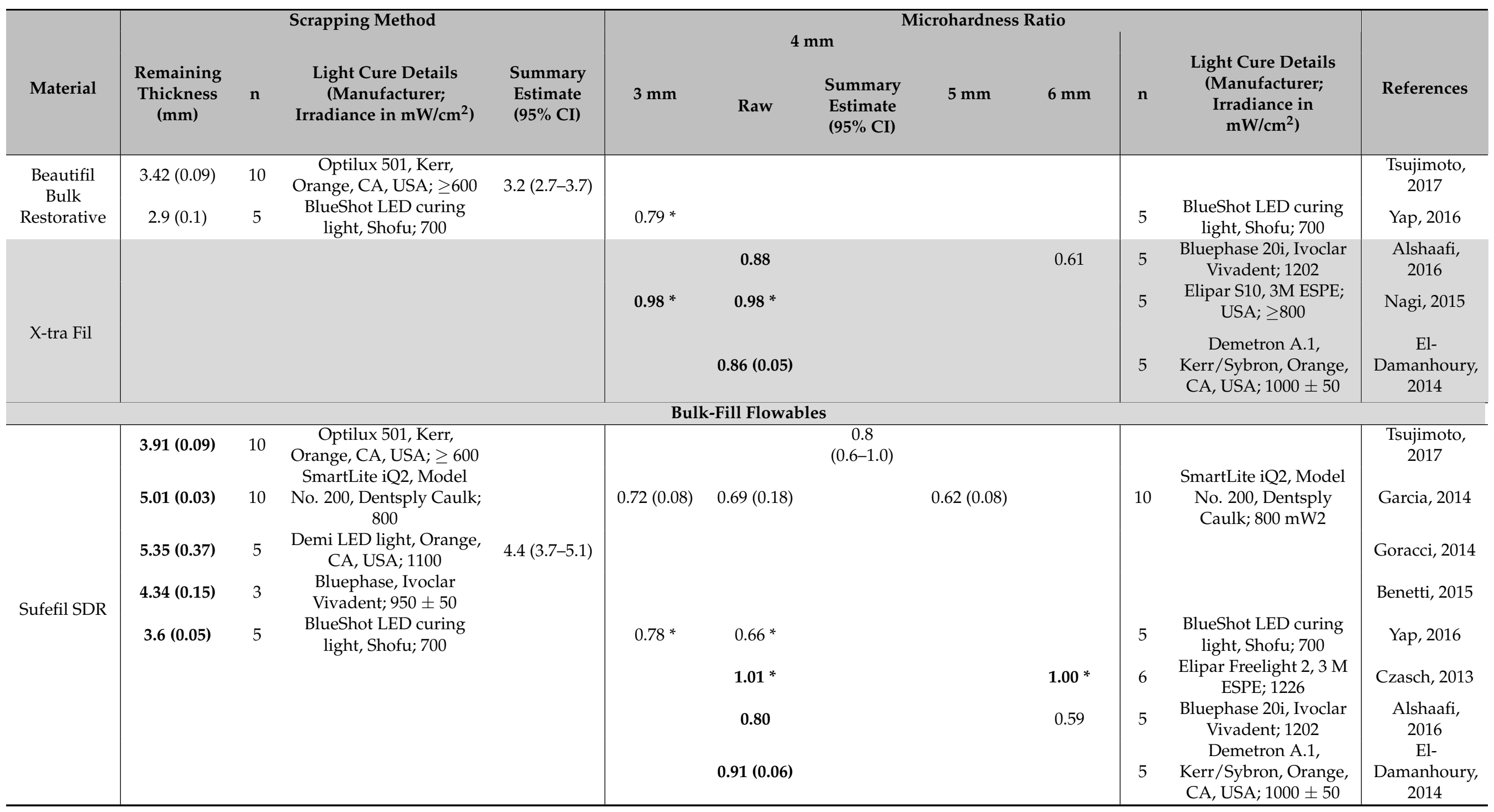


Table 6. Cont.

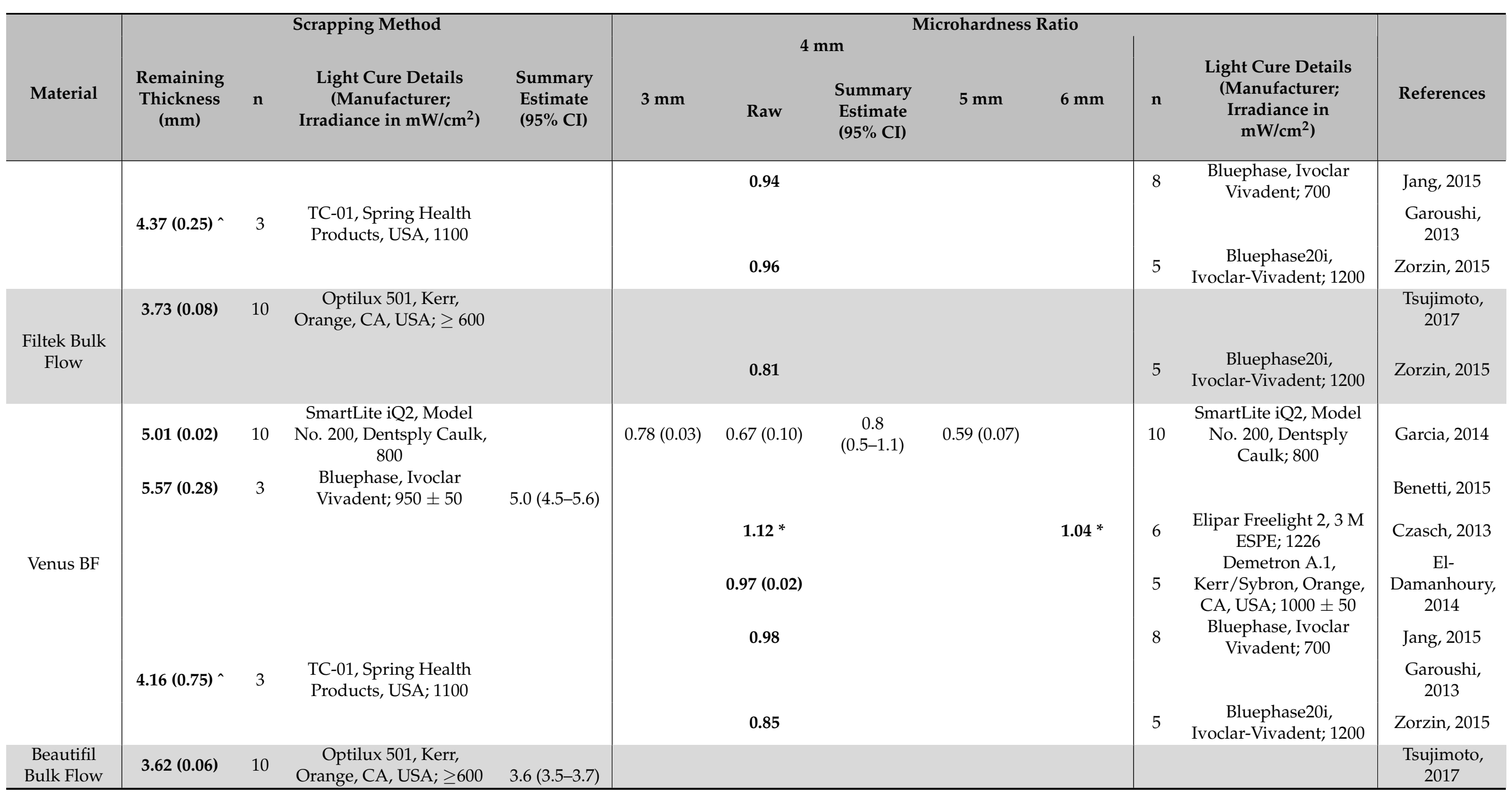


Table 6. Cont.

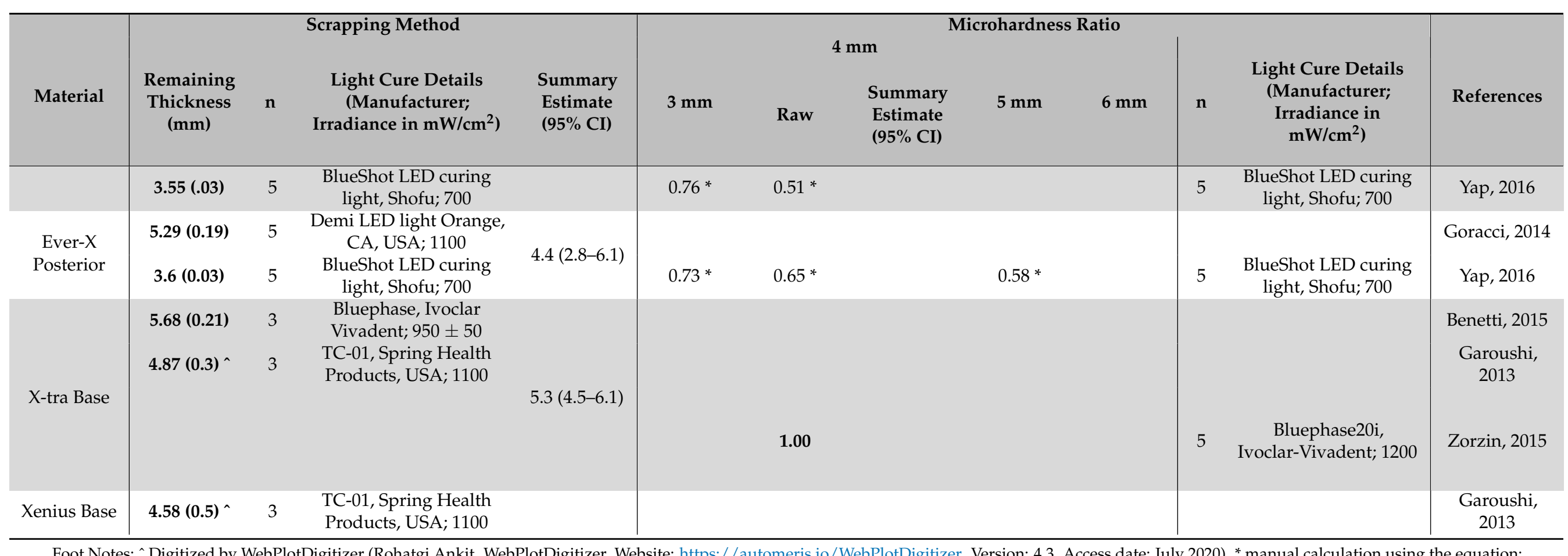

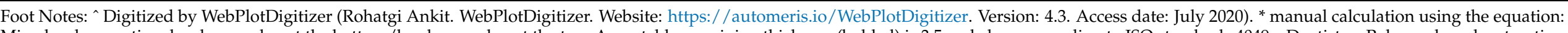

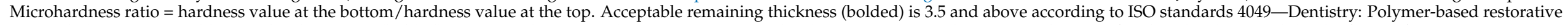

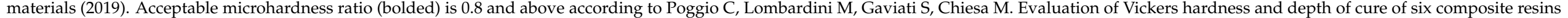
photo-activated with different polymerization methods. J. Conserv. Dent. 2012, 15, 237-241. 
Estimates of the DoC, remaining thickness, and MHR for all materials are presented in Figure 2. Summary estimates are presented, when available; and when they were not, individual study estimates are presented.

a)

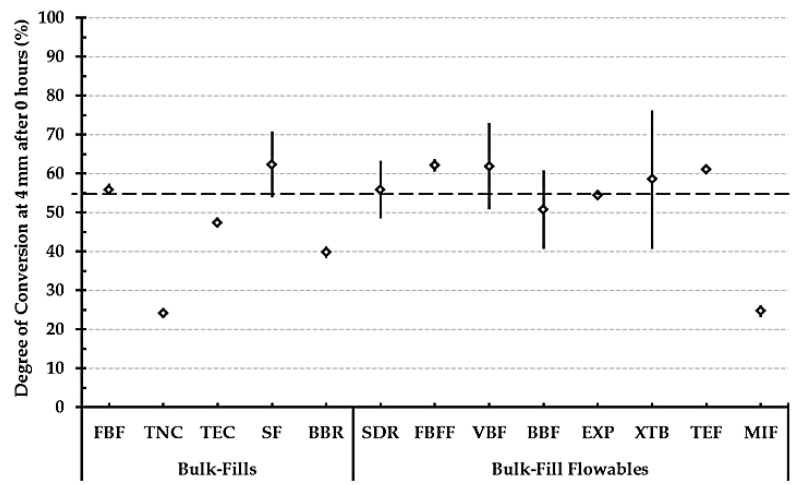

c)

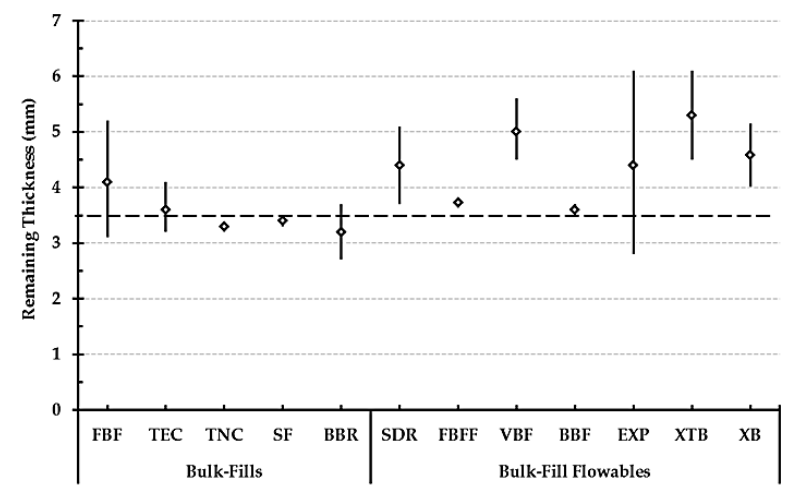

b)

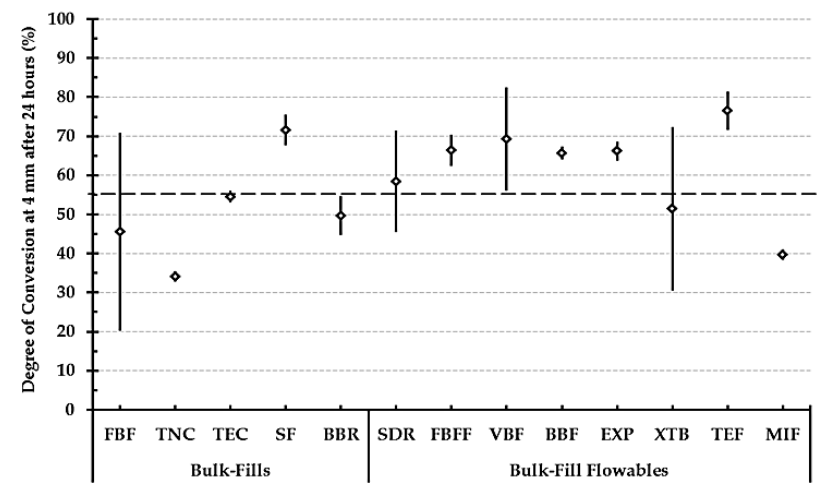

d)

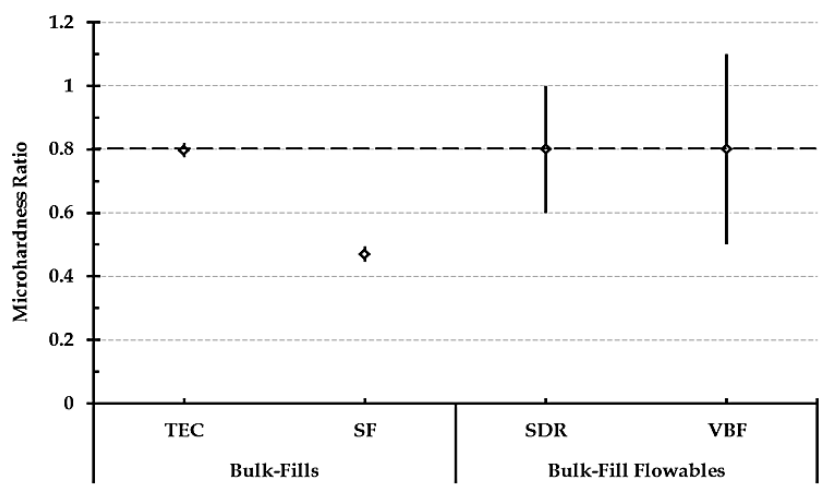

Figure 2. Graphs showing mean values of study parameters from publications reporting means and standard deviations. Summary estimates from relevant meta-analysis were included when performed: (a) degree of conversion at $4 \mathrm{~mm}(0 \mathrm{~h}$ after curing), (b) degree of conversion at $4 \mathrm{~mm}$ (24 h after curing), (c) remaining thickness after scrapping method, and (d) microhardness ratio at $4 \mathrm{~mm}$ ( $24 \mathrm{~h}$ after curing). Diamonds represent means of individual studies or summary estimates of meta-analysis. Black bars represent 95\% CI. Dashed line represents acceptable value for the respective parameter. FBF: Filtek Bulk-Fill, TNC: Tetric N-Ceram Bulk Fill, TEC: Tetric Evo-Ceram Bulk-Fill, SF: Sonic Fill, BBR: Beautifil Bulk Restorative, SDR: SureFil SDR, FBFF: Filtek Bulk-Fill Flowable, VBF: Venus Bulk-Fill, BBF: Beautifil Bulk Flow, EXP: Ever-X Posterior, XTB: X-tra Base, TEF: Tetric Evo-Flow Bulk-Fill, MIF: MI Fil, XB: Xenius Base.

Meta-analyses of the outcome values were performed in 15 of the studies [30-32,3438,40-42,44-47]; which had estimates for the chosen fixed parameters in two studies or more (Tables 5 and 6).

\section{Discussion}

Numerous studies in the literature explored and compared the polymerization efficiency either directly or indirectly to ensure favorable clinical service. The results of these studies were inconsistent which makes it crucial to examine the current available evidence regarding BFCs in relation to their DC and DoC. The aim of this study was to investigate the polymerization efficiency of BFCs according to the available evidence in the published literature.

Of the indirect methods studies, SM differs from the MHR method in that the first is a subjective method where inter-operator differences can affect the reliability of the results, while the latter is a quantitative method which makes its results more objective and reliable. In both of these tests, the DC is measured indirectly by measuring the hardness values of the resin composite. On the other hand, the direct methods (RS and FTIR) measure the actual amounts of converted monomers by detecting the ratio between double and single bonds within the polymer chain. 
With regards to irradiance values of light curing, the recommended values for BF materials is more than $1000 \mathrm{~mW} / \mathrm{cm}^{2}$ [30]. Not all studies performed light-curing using devices with the minimal required irradiance [34,37,39,40,45,49] According to the reported results by the authors, the clinically acceptable values were not related to the recommended light-cure irradiance as some studies reported unacceptable DoC $[37,38,40]$ and DC $[32,35,41,42,46,47]$ values with $\geq 1000 \mathrm{~mW} / \mathrm{cm}^{2}$ irradiance and some studies reported acceptable DoC $[34,37,40,45]$ values with $<1000 \mathrm{~mW} / \mathrm{cm}^{2}$ irradiance. Of course, these values can be affected greatly with duration of light-curing exposure.

\subsection{Data Extraction and Summary Estimates}

Some studies had the results values illustrated on graphs and were not stated in tables neither within the text. These studies were: Lempel 2016 for the DC values [46] and Garouchi 2013 for the remaining thickness values [38]. Still, the values were extracted from the graphs using Web digitizer software [51]. Furthermore, some studies did not report the MHR and only reported the top and bottom microhardness values. These studies were Nagi 2015; Yap, 2016 and Czasch, 2013 [36,45,49]. In these instances, MHRs were calculated manually from the reported top and bottom values of these investigations. In the studies with manually calculated MHRs, the standard deviations of the mean MHRs could not be calculated. Other studies reported the mean MHRs without the standard deviations and these studies were Alshaafi 2016; Jang, 2015 and Zorzin, 2015 [33,39,42]. The authors were contacted for the missing information; however, neither SD values nor raw data were obtained. Thus, these studies were excluded from the meta-analyses while been considered for qualitative analysis.

Summary estimates of the DC results were calculated for materials that were assessed in more than one study. While materials assessed in one study, only the study estimate was presented. Materials where data extraction was done from a single study were Filtek Bulk-Fill (at 0 h), Tetric N-Ceram Bulk Fill, Tetric Evo-Ceram Bulk-Fill (at top and $6 \mathrm{~mm}$ depths), Sonic Fill (at top), Beautifil Bulk Restorative (at top, $6 \mathrm{~mm}$ depth at $0 \mathrm{~h}$, and $4 \mathrm{~mm}$ depth at 24 h), Filtek Bulk-Fill Flowable, Venus Bulk-Fill (at $6 \mathrm{~mm}$ depth), Beautifil Bulk Flow (at $6 \mathrm{~mm}$ depth), X-tra base (at top at $0 \mathrm{~h}$ ), Tetric Evo-Flow Bulk-Fill, and MI Fil. Not all materials were tested at the two time points $(0$ and $24 \mathrm{~h})$ and not all materials were tested at all depths (top, $4 \mathrm{~mm}$ and $6 \mathrm{~mm}$ ). The only two materials which have DC values at all time points and depths are SureFill SDR and Venus Bulk-Fill.

\subsection{Degree of Conversion Data}

From the DC data in Table 5, it was noticed that some materials did not have an acceptable DC values even at the top surface: Filtek bulk-fill $(24 \mathrm{~h})$, Tetric N-Ceram bulk fill (0 and $24 \mathrm{~h})$, Tetric Evo Ceram bulk-fill ( 0 and $24 \mathrm{~h})$, Beautifil Bulk restorative (0 and $24 \mathrm{~h}$ ), and MI Fil (0 $\mathrm{h}$ and $24 \mathrm{~h}$ at $4 \mathrm{~mm})$. Of these materials, only one reported acceptable top surface DC value after $24 \mathrm{~h}$ (MI Fil). It was also noticed that the only regular BFC with acceptable DC values at all depths and time points is Sonic Fill. On the other hand, values reported by BFFs (except X-tra Base and MI Fill) were acceptable at almost all depths and time points.

Upon analyzing the DC values comparing the $0 \mathrm{~h}$ and $24 \mathrm{~h}$ data, it was noticed that some studies reported a lower DC value at $24 \mathrm{~h}$ than at $0 \mathrm{~h}$, which was not in accordance with scientific knowledge that the polymerization process continues after light curing [52,53], thus expecting higher DC values with time. This reduction in the DC values with time is considered small and insignificant at the top surface for Filtek Bulk-Fill Flowable and X-tra Base, but is considered significant at the $4 \mathrm{~mm}$ depth values for X-tra base and Regular Filtek Bulk-fill where the values becomes unacceptable after $24 \mathrm{~h}$. The latter might be explained by the fact that the $24 \mathrm{~h}$ values for $\mathrm{X}$-tra base and Regular Filtek Bulk-fill were calculated as summary estimates from multiple studies who reported significantly different values. 
Some materials were associated with higher DC values at increasing depths which was not in accordance with the scientific knowledge that deeper surfaces receive less curing light intensity. SureFil SDR at $0 \mathrm{~h}$ showed the highest DC value at the top (61.1\%) but the $6 \mathrm{~mm}$ depth value (56.4\%) was slightly higher than the $4 \mathrm{~mm}$ depth value (55.8\%). Beautifil Bulk Flow showed the same observation with the highest DC value at the top $(57.3 \%)$ but the $6 \mathrm{~mm}$ depth value $(55.0 \%)$ was significantly higher than the none acceptable $4 \mathrm{~mm}$ depth value $(50.7 \%)$. Venus Bulk-Fill reported the similar observation at $0 \mathrm{~h}$ with the highest DC values at $6 \mathrm{~mm}$ depth $(65.6 \%)$ then at $4 \mathrm{~mm}$ depth $(61.8 \%)$ and then at the top $(61.3 \%)$. Venus Bulk-Fill reported a DC value at $4 \mathrm{~mm}$ depth $(69.3 \%)$ higher than at the top $(68.1 \%)$ at $24 \mathrm{~h}$. Filtek Bulk-Fill Flowable reported a slightly higher value at the $4 \mathrm{~mm}$ depth $(66.4 \%)$ than the top surface $(65.9 \%)$ at $24 \mathrm{~h}$. This can be explained because some results were calculated as summary estimates from multiple studies reporting significantly different values. For results from single studies, this observation maybe caused by the type of background on which the materials were light cured against and the possibility of a reflective surface being used on the base of mold [31,32,36,42]. Light curing on reflective surfaces might contribute to increasing the DC at the bottom surface since the deeper layers will receive curing light from both the original and the reflected light.

From the analysis of these data, it was found that the BFFs resin composites performed better than the regular BFCs with more acceptable DC values at all time points and depths. This could be explained by the lower filler content which allows for better penetration of light at deeper depths in addition to the higher degree of conversion values at the top surface due to higher ratio of the resin to filler [54].

Among the 21 studies found, one study did not follow the protocol for DC. Li et al., 2015 [43] reported the curing profile of four BFCs (everX Posterior, Filtek Bulk Fill Flowable, SDR, and Tetric EvoCeram Bulk Fill) using RS. The authors found that ever-X Posterior and Filtek Bulk Fill Flowable were effectively cured with DC equal to at least $72 \%$ (90\% of the mean maximum DC) at depths of 5.47 and $7.84 \mathrm{~mm}$ respectively. SDR was effectively cured with DC equal to at least $69 \%$ (90\% of the mean maximum DC) at a depth of $9.45 \mathrm{~mm}$. Tetric EvoCeram Bulk Fill was effectively cured with DC equal to at least $64 \%(90 \%$ of the mean maximum DC) at a depth of $3.14 \mathrm{~mm}$. Three BFCs in this study, can be compared to the findings of the extracted data from the acceptable studies in this systematic review but none of them had consistent findings as the effective DC reported by Li et al. 2015 were at deeper depths compared to the ones reported by the other studies [43]. One explanation could be that the unreported light curing distance in the included studies which was tested in the paper by Li and collaborators who concluded that the light-cure position has less critical effect on the BFCs' DC compared to conventional RBC [43]. Other investigator-dependent and technical variables (e.g., light cure intensity and resin composite shade) can add up to the light curing distance and might result in reported variation in DC values and DoC.

\subsection{Depth of Cure Data}

DoC results for the studies with the fixed parameters were extracted from the acceptable values and the summary estimates of the meta-analysis were calculated for the materials with more than one study results. Materials where their data were extracted from a single study only for the SM were Tetric N-Ceram Bulk-Fill, Filtek Bulk Flow and Xenius base. For the MHR, due to the missing standard deviation values in several studies [33,36,39,42,45,49]; summary estimate values could only be calculated for materials with at least two studies having the mean and standard deviation values. The only two materials we were able to have a summary estimate for from two studies for each are SureFil SDR and Venus Bulk-Fill both at 4 mm depth (Garcia, 2014 and El-Damanhoury, 2014) [30,40] and the rest of the materials had either single study (Tetric Evo-Ceram Bulk-Fill at 3 and $6 \mathrm{~mm}$, SonicFill at $6 \mathrm{~mm}$, Beautifil Bulk Restorative, X-tra fil at 3 and $6 \mathrm{~mm}$, SureFil SDR at $5 \mathrm{~mm}$, Filtek Bulk Flow, Venus Bulk-Fill at 3, 5, and $6 \mathrm{~mm}$, Beautifil Bulk Flow, Ever-X Posterior and X-tra base) or more than one study that could not calculate summary estimates for them due to missing standard deviation (Tetric Evo-Ceram Bulk-Fill at $4 \mathrm{~mm}$, Tetric 
$\mathrm{N}$-Ceram Bulk-Fill at $4 \mathrm{~mm}$, SonicFill at $4 \mathrm{~mm}$, X-tra fil at $4 \mathrm{~mm}$, and SureFil SDR at 3 and $6 \mathrm{~mm})$.

For the SM, all BFF materials had acceptable values with each single study and with the summary estimates. On the other hand, only two regular BFCs had acceptable values: Filtek Bulk-Fill and Tetric Evo-Ceram Bulk-Fill while Tetric N-Ceram Bulk-Fill, Sonic Fill and Beautifil Bulk Restorative had unacceptable values with each single study and with the summary estimates but the numbers were close to the acceptable value (Figure 2c).

For the MHR, all of the listed materials have reported MHRs at $3 \mathrm{~mm}$ depth except Filtek Bulk Flow and X-tra base. Of these materials only two reported acceptable MHRs at $3 \mathrm{~mm}$ depth (Tetric Evo-Ceram Bulk-Fill and X-tra fil). At $4 \mathrm{~mm}$ depth all materials reported MHR except Beautifil Bulk Restorative. If only data with SD together with the summary estimates were considered, materials with acceptable MHRs would be: Tetric Evo-Ceram Bulk-Fill, X-tra fil, SureFil SDR and Venus Bulk-Fill. If we considered numbers without SD, it will not be possible to reach a consensus with some materials on whether we consider the reported MHR values acceptable or not. This is because studies' results are not consistent and both acceptable and unacceptable values were reported for the same material of Tetric Evo-Ceram Bulk-Fill, SureFil SDR and Venus Bulk-Fill. The rest of the materials had either consistent findings or were reported by a single study and the materials with acceptable values were X-tra fil and X-tra base. At $5 \mathrm{~mm}$ depth, only four materials reported MHRs from single studies and they all had unacceptable values. These materials were SonicFill, SureFil SDR, Venus Bulk-Fill and Ever-X Posterior. While, at $6 \mathrm{~mm}$ depth, only five materials reported MHRs four of them were reported from single studies of which Venus Bulk-fill was the only material with acceptable value and SureFil SDR reported MHRs from two studies with an acceptable value by one study [36] and unacceptable value by another study [33]. It is clear from the MHR values that increasing the depth reduced the values but more homogenous studies reporting the required data with SD values at each depth are needed to be able to reach a more solid conclusion.

\subsection{Strengths and Limitations}

This systematic review has several strengths; it is the first investigation compiling the results from the four methods used to assess polymerization efficiency. Further, standardized outcomes were presented in a concise manner corresponding to the relevant clinical procedure allowing for meaningful statistical synthesis and interpretation of the results. Another strength is that when missing data were encountered, authors were contacted for the relevant data, a dedicated software was used to depict values from figures, or manually calculated by the investigators. Lastly, a rigorous methodology was utilized where two independent reviewers performed all steps of the review which were then overseen by all authors.

Although within our search, a recent systematic review by Lima and collaborators [55] was identified, several key differences between that article compared to the current investigation can be identified. First, in the current investigation, papers published until January 2019 were included and analyzed, which is one and half years after the included search timeframe of the systematic review by Lima and colleagues [55]. Given the rapid advancement in BF composite formulations, the inclusion of recent publications is beneficiary to the researchers and clinicians in order to guide future research efforts and clinical protocols. Additionally, all tests denoting DC either directly or indirectly were included which were complemented by a meta-analysis with heterogeneity assessment. Furthermore, in the current systematic review, parameters were standardized in order to facilitate combining summary estimates into a meta-analysis which gives more optimum quantitative analysis of the data. Lastly, the tables of the current investigation collected and processed the data reported in the studies in an easy to follow manner enabling easier visualization means and standard deviations for each material and testing method reported by each cited paper.

Several factors could have influenced our meta-analyses summary estimates; the number of studies assessing each type of material was relatively small, and there was 
marked statistical heterogeneity between the studies. However, heterogeneity estimates are not accurate when the number of studies assessed is small [56]. Furthermore, given the controlled environment in laboratory studies, and that we fixed certain parameters (depth and light curing time) for estimates that were statistically synthesized, and given that a random effects model was used; we believe that these factors make our summary estimates interpretable. Furthermore, the tool used for quality assessment was not validated, as we preferred to use a list of questions that were tailored to our research inquiry. Still, we believe that risk of bias would not be a major concern as all the studies included are in vitro studies which are done in a controlled laboratory environments following previously published well known methodological protocols [22,24,26]. Additionally, all outcomes were assessed objectively using numerical values output from relevant devices.

\section{Conclusions}

From the above analysis of the data from the reported and selected studies, it is evident that not all reported BFCs showed results that are in accordance with their manufacturer's claims, though there is not enough evidence to reach a solid conclusion. It was also evident that BFFs have higher and more acceptable DoC and DC values compared to regular BFCs. There was no direct relation found between the light-cure irradiance and the outcome. Increasing depth was clearly affecting the outcome and reducing the efficiency of polymerization for most of the data and other data with more DoC at the bottom surface could be the matter of the type of background against which the materials were light cured which can be investigated in future studies. Many limitations were found that affected the decision and resulted in lack of strong evidence to reach consensus. These limitations were the lack of homogenous studies that measured the DoC and/or DC at standard time points, depths, light-curing irradiance, and light curing times. Additionally, the missing data for sufficient statistical analysis and they were not available after contacting the authors. It is suggested that future studies be carried out using a standard methodology following the ISO 4049 standard and manufacturer's instructions regarding the curing time and irradiance and reporting complete data at all time points and depths ensuring stronger evidence with increased potential for reaching consensus. In addition, researchers can refer to the work by Ferracane, Ilie, and collaborators for recent testing guidance $[57,58]$.

Author Contributions: Conceptualization, R.A.A.; methodology, R.A.A., N.J.F. and H.M.N.; software, N.J.F.; validation, R.A.A., N.J.F. and H.M.N.; formal analysis, R.A.A., N.J.F. and H.M.N.; investigation, L.A., N.N. and R.G.; resources, L.A., N.N. and R.G.; data curation, R.A.A., N.J.F. and H.M.N.; writing-original draft, L.A., N.N. and R.G.; writing—review and editing, R.A.A., N.J.F. and H.M.N.; visualization, L.A., N.N. and R.G.; supervision, R.A.A., N.J.F. and H.M.N. All authors have read and agreed to the published version of the manuscript.

Funding: This research received no external funding.

Conflicts of Interest: The authors declare no conflict of interest.

\section{References}

1. Demarco, F.F.; Corrêa, M.B.; Cenci, M.; Moraes, R.R.; Opdam, N.J. Longevity of posterior composite restorations: Not only a matter of materials. Dent. Mater. 2012, 28, 87-101. [CrossRef] [PubMed]

2. Chesterman, J.; Jowett, A.; Gallacher, A.; Nixon, P. Bulk-fill resin-based composite restorative materials: A review. Br. Dent. J. 2017, 222, 337-344. [CrossRef] [PubMed]

3. Poggio, C.; Lombardini, M.; Gaviati, S.; Chiesa, M. Evaluation of Vickers hardness and depth of cure of six composite resins photo-activated with different polymerization modes. J. Conserv. Dent. 2012, 15, 237-241. [CrossRef] [PubMed]

4. Reis, A.F.; Vestphal, M.; Amaral, R.C.D.; Rodrigues, J.A.; Roulet, J.-F.; Roscoe, M.G. Efficiency of polymerization of bulk-fill composite resins: A systematic review. Braz. Oral Res. 2017, 31, e59. [CrossRef]

5. Rees, J.S.; Jagger, D.C.; Williams, D.R.; Brown, G.; Duguid, W. A reappraisal of the incremental packing technique for light cured composite resins. J. Oral Rehabil. 2004, 31, 81-84. [CrossRef]

6. Ferracane, J.L. Placing Dental Composites-A Stressful Experience. Oper. Dent. 2008, 33, 247-257. [CrossRef] [PubMed]

7. Braga, R.R.; Ballester, R.Y.; Ferracane, J.L. Factors involved in the development of polymerization shrinkage stress in resincomposites: A systematic review. Dent. Mater. 2005, 21, 962-970. [CrossRef] 
8. Vandewalker, J.P.; Casey, J.A.; Lincoln, T.A.; Vandewalle, K.S. Properties of dual-cure, bulk-fill composite resin restorative materials. Gen. Dent. 2016, 64, 68-73. [PubMed]

9. Van Ende, A.; De Munck, J.; Lise, D.P.; Van Meerbeek, B. Bulk-Fill Composites: A Review of the Current Literature. J. Adhes Dent. 2017, 19, 95-109.

10. Menees, T.S.; Lin, C.P.; Kojic, D.D.; Burgess, J.O.; Lawson, N.C. Depth of cure of bulk fill composites with monowave and polywave curing lights. Am. J. Dent. 2015, 28,357-361.

11. Kim, E.-H.; Jung, K.-H.; Son, S.-A.; Hur, B.; Kwon, Y.-H.; Park, J.-K. Effect of resin thickness on the microhardness and optical properties of bulk-fill resin composites. Restor. Dent. Endod. 2015, 40, 128-135. [CrossRef]

12. Monterubbianesi, R.; Orsini, G.; Tosi, G.; Conti, C.; Librando, V.; Procaccini, M.; Putignano, A. Spectroscopic and Mechanical Properties of a New Generation of Bulk Fill Composites. Front. Physiol. 2016, 7, 652. [CrossRef]

13. Bucuta, S.; Ilie, N. Light transmittance and micro-mechanical properties of bulk fill vs. conventional resin based composites. Clin. Oral Investig. 2014, 18, 1991-2000. [CrossRef] [PubMed]

14. Correa, M.B.; Henn, S.; Marimon, J.L.M.; Rodrigues, S.A.; Demarco, F.F. Factors influencing the microhardness of a microhybrid composite. Gen. Dent. 2010, 58, 94-98.

15. Estafan, D.; Agosta, C. Eliminating microleakage from the composite resin system. Gen. Dent. 2004, 51, 506-509.

16. Rastelli, A.N.S.; Jacomassi, D.P.; Bagnato, V.S. Degree of conversion and temperature increase of a composite resin light cured with an argon laser and blue LED. Laser Phys. 2008, 18, 1570-1575. [CrossRef]

17. Moraes, L.G.; Unesp, P.; Rocha, R.S.; Unesp, F.; Menegazzo, L.M.; Araujo, E.B.; Yukimito, K.; Moraes, J.C.S. Infrared spectroscopy: A tool for determination of the degree of conversion in dental composites. J. Appl. Oral Sci. 2008, 16, 145-149. [CrossRef]

18. Amirouche-Korichi, A.; Mouzali, M.; Watts, D.C. Effects of monomer ratios and highly radiopaque fillers on degree of conversion and shrinkage-strain of dental resin composites. Dent. Mater. 2009, 25, 1411-1418. [CrossRef]

19. Ilie, N.; Keßler, A.; Durner, J. Influence of various irradiation processes on the mechanical properties and polymerisation kinetics of bulk-fill resin based composites. J. Dent. 2013, 41, 695-702. [CrossRef]

20. Aravamudhan, K.; Floyd, C.J.; Rakowski, D.; Flaim, G.; Dickens, S.H.; Eichmiller, F.C.; Fan, P. Light-emitting diode curing light irradiance and polymerization of resin-based composite. J. Am. Dent. Assoc. 2006, 137, 213-223. [CrossRef]

21. Alshali, R.Z.; Silikas, N.; Satterthwaite, J.D. Degree of conversion of bulk-fill compared to conventional resin-composites at two time intervals. Dent. Mater. 2013, 29, e213-e217. [CrossRef]

22. Bouschlicher, M.R.; Rueggeberg, F.A.; Wilson, B.M. Correlation of bottom-to-top surface microhardness and conversion ratios for a variety of resin composite compositions. Oper. Dent. 2005, 29, 698-704.

23. Lloyd, C.H.; Scrimgeour, S.N.; Chudek, J.A.; Mackay, R.L.; Hunter, G.; Pananakis, D.; Abel, E.W. Determination of the depth of cure for VLC composites by nuclear magnetic resonance microimaging. Dent. Mater. 1994, 10, 128-133. [CrossRef]

24. Flury, S.; Hayoz, S.; Peutzfeldt, A.; Hüsler, J.; Lussi, A. Depth of cure of resin composites: Is the ISO 4049 method suitable for bulk fill materials? Dent. Mater. 2012, 28, 521-528. [CrossRef] [PubMed]

25. Dewald, J.; Ferracane, J. A Comparison of Four Modes of Evaluating Depth of Cure of Light-activated Composites. J. Dent. Res. 1987, 66, 727-730. [CrossRef] [PubMed]

26. Moore, B.K.; Platt, J.A.; Borges, G.; Chu, T.-M.G.; Katsilieri, I. Depth of Cure of Dental Resin Composites: ISO 4049 Depth and Microhardness of Types of Materials and Shades. Oper. Dent. 2008, 33, 408-412. [CrossRef]

27. Moher, D.; Liberati, A.; Tetzlaff, J.; Altman, D.G.; The PRISMA Group. Preferred reporting items for systematic reviews and meta-analyses: The PRISMA statement. PLoS Med. 2009, 6, e1000097. [CrossRef]

28. International Organization for Standardization. ISO-4049. In Dentistry-Polymer-Based Restorative Materials; ISO: Geneva, Switzerland, 2019.

29. Higgins, J.P.T.; Thompson, S.G. Quantifying heterogeneity in a meta-analysis. Stat. Med. 2002, 21, 1539-1558. [CrossRef] [PubMed]

30. El-Damanhoury, H.; Platt, J.A. Polymerization Shrinkage Stress Kinetics and Related Properties of Bulk-fill Resin Composites. Oper. Dent. 2014, 39, 374-382. [CrossRef] [PubMed]

31. Marovic, D.; Tauböck, T.T.; Attin, T.; Panduric, V.; Tarle, Z. Monomer conversion and shrinkage force kinetics of low-viscosity bulk-fill resin composites. Acta Odontol. Scand. 2015, 73, 474-480. [CrossRef]

32. Ilie, N.; Fleming, G.J. In vitro comparison of polymerisation kinetics and the micro-mechanical properties of low and high viscosity giomers and RBC materials. J. Dent. 2015, 43, 814-822. [CrossRef] [PubMed]

33. Alshaafi, M.M.; Haenel, T.; Sullivan, B.; Labrie, D.; Alqahtani, M.Q.; Price, R.B. Effect of a broad-spectrum LED curing light on the Knoop microhardness of four posterior resin based composites at 2, 4 and 6-mm depths. J. Dent. 2016, 45, 14-18. [CrossRef]

34. Tsujimoto, A.; Barkmeier, W.W.; Takamizawa, T.; Latta, M.A.; Miyazaki, M. Depth of cure, flexural properties and volumetric shrinkage of low and high viscosity bulk-fill giomers and resin composites. Dent. Mater. J. 2017, 36, 205-213. [CrossRef] [PubMed]

35. Al-Ahdal, K.; Ilie, N.; Silikas, N.; Watts, D.C. Polymerization kinetics and impact of post polymerization on the Degree of Conversion of bulk-fill resin-composite at clinically relevant depth. Dent. Mater. 2015, 31, 1207-1213. [CrossRef] [PubMed]

36. Czasch, P.; Ilie, N. In vitro comparison of mechanical properties and degree of cure of bulk fill composites. Clin. Oral Investig. 2012, 17, 227-235. [CrossRef] [PubMed]

37. Benetti, A.R.; Havndrup-Pedersen, C.; Honoré, D.; Pedersen, M.K.; Pallesen, U. Bulk-Fill Resin Composites: Polymerization Contraction, Depth of Cure, and Gap Formation. Oper. Dent. 2015, 40, 190-200. [CrossRef] [PubMed] 
38. Garoushi, S.; Säilynoja, E.; Vallittu, P.K.; Lassila, L. Physical properties and depth of cure of a new short fiber reinforced composite. Dent. Mater. 2013, 29, 835-841. [CrossRef] [PubMed]

39. Jang, J.-H.; Park, S.-H.; Hwang, I.-N. Polymerization Shrinkage and Depth of Cure of Bulk-Fill Resin Composites and Highly Filled Flowable Resin. Oper. Dent. 2015, 40, 172-180. [CrossRef]

40. Garcia, D.; Yaman, P.; Dennison, J.; Neiva, G. Polymerization Shrinkage and Depth of Cure of Bulk Fill Flowable Composite Resins. Oper. Dent. 2014, 39, 441-448. [CrossRef]

41. Goracci, C.; Cadenaro, M.; Fontanive, L.; Giangrosso, G.; Juloski, J.; Vichi, A.; Ferrari, M. Polymerization efficiency and flexural strength of low-stress restorative composites. Dent. Mater. 2014, 30, 688-694. [CrossRef]

42. Zorzin, J.; Maier, E.; Harre, S.; Fey, T.; Belli, R.; Lohbauer, U.; Petschelt, A.; Taschner, M. Bulk-fill resin composites: Polymerization properties and extended light curing. Dent. Mater. 2015, 31, 293-301. [CrossRef]

43. Li, X.; Pongprueksa, P.; Van Meerbeek, B.; De Munck, J. Curing profile of bulk-fill resin-based composites. J. Dent. 2015, 43, 664-672. [CrossRef] [PubMed]

44. Par, M.; Lapas-Barisic, M.; Gamulin, O.; Panduric, V.; Spanovic, N.; Tarle, Z. Long Term Degree of Conversion of two Bulk-Fill Composites. Acta Stomatol. Croat. 2016, 50, 292-300. [CrossRef] [PubMed]

45. Yap, A.U.J.; Pandya, M.; Toh, W.S. Depth of cure of contemporary bulk-fill resin-based composites. Dent. Mater. J. 2016, 35, 503-510. [CrossRef] [PubMed]

46. Lempel, Z.; Czibulya, B.; Kovács, J.; Szalma, Á.; Tóth, S.; Kunsági-Máté, Z.; Varga, K.; Böddi, E. Degree of Conversion and BisGMA, TEGDMA, UDMA Elution from Flowable Bulk Fill Composites. Int. J. Mol. Sci. 2016, 17, 732. [CrossRef]

47. Jain, L.; Mehta, D.; Meena, N.; Gupta, R. Influence of light energy density, composite type, composite thickness, and postcuring phase on degree of conversion of bulk-fill composites. Contemp. Clin. Dent. 2018, 9, S147-S152. [CrossRef]

48. Gonçalves, F.; Campos, L.M.D.P.; Rodrigues-Júnior, E.C.; Costa, F.V.; Marques, P.A.; Francci, C.E.; Braga, R.R.; Boaro, L.C.C. A comparative study of bulk-fill composites: Degree of conversion, post-gel shrinkage and cytotoxicity. Braz. Oral Res. 2018, 32, e17. [CrossRef]

49. Nagi, S.M.; Moharam, L.M.; Zaazou, M.H. Effect of resin thickness, and curing time on the micro-hardness of bulk-fill resin composites. J. Clin. Exp. Dent. 2015, 7, e600-e604. [CrossRef]

50. Pongprueksa, P.; De Munck, J.; Duca, R.C.; Poels, K.; Covaci, A.; Hoet, P.; Godderis, L.; Van Meerbeek, B.; Van Landuyt, K.L. Monomer elution in relation to degree of conversion for different types of composite. J. Dent. 2015, 43, 1448-1455. [CrossRef]

51. Rohatgi, A. Web Plot Digitizer. Version: 4. Available online: https://automeris.io/WebPlotDigitizer (accessed on 1 August 2020).

52. Truffier-Boutry, D.; Demoustier-Champagne, S.; Devaux, J.; Biebuyck, J.-J.; Mestdagh, M.; Larbanois, P.; Leloup, G. A physicochemical explanation of the post-polymerization shrinkage in dental resins. Dent. Mater. 2006, 22, 405-412. [CrossRef]

53. Leprince, W.M.; Palin, M.A.; Hadis, J.; Devaux, G.; Leloup, J.G. Progress in dimethacrylate-based dental composite technology and curing efficiency. Dent. Mater. 2013, 29, 139-156. [CrossRef] [PubMed]

54. Halvorson, R.H.; Erickson, R.L.; Davidson, C.L. The effect of filler and silane content on conversion of resin-based composite. Dent. Mater. 2003, 19, 327-333. [CrossRef]

55. Lima, R.B.W.; Troconis, C.C.M.; Moreno, M.B.P.; Murillo-Gómez, F.; De Goes, M.F. Depth of cure of bulk fill resin composites: A systematic review. J. Esthet. Restor. Dent. 2018, 30, 492-501. [CrossRef] [PubMed]

56. von Hippel, P.T. The heterogeneity statistic I2 can be biased in small meta-analyses. BMC Med. Res. Methodol. 2015, 15, 35. [CrossRef]

57. Ilie, N.; Hilton, T.; Heintze, S.; Hickel, R.; Watts, D.; Silikas, N.; Stansbury, J.; Cadenaro, M.; Ferracane, J. Academy of Dental Materials guidance-Resin composites: Part I-Mechanical properties. Dent. Mater. 2017, 33, 880-894. [CrossRef]

58. Ferracane, J.; Hilton, T.; Stansbury, J.; Watts, D.; Silikas, N.; Ilie, N.; Heintze, S.; Cadenaro, M.; Hickel, R. Academy of Dental Materials guidance-Resin composites: Part II-Technique sensitivity (handling, polymerization, dimensional changes). Dent. Mater. 2017, 33, 1171-1191. [CrossRef] [PubMed] 